\title{
Multi-Objective Optimization of Tension Leg Platform Using Evolutionary Algorithm Based on Surrogate Model
}

\author{
Xinshu Zhang ${ }^{a, b, *}$ Xingyu Song ${ }^{a, b}$, Wenzhen Qiu ${ }^{a, b}$, Zhiming Yuan ${ }^{c}$, Yunxiang You ${ }^{a, b}$, Naiming \\ Deng $^{a, b}$ \\ ${ }^{a}$ State Key Laboratory of Ocean Engineering, Shanghai Jiao Tong University, \\ Shanghai, 200240, China \\ ${ }^{b}$ Collaborative Innovation Center for Advanced Ship and Deep-Sea Exploration (CISSE), \\ Shanghai, 200240, China \\ ${ }^{c}$ Department of Naval Architecture, Ocean and Marine Engineering, University of Strathclyde, \\ Glasgow, G4 OLZ, UK
}

\begin{abstract}
An Innovative Tension Leg Platform (TLP) Optimization Program, called ITOP, has been developed to solve the multi-objective optimization problem for TLP. We first examine the hydrodynamic behavior of a base TLP for wave headings between $0^{\circ}$ and $45^{\circ}$. The numerical results show that the maximum heave and surge motion responses occur in $0^{\circ}$ wave heading in long-crest waves. It is found that the dynamic tension of No. 8 tendon is larger than the other tendons and reaches its maximum in $45^{\circ}$ wave heading. It can be attributed to the fact that heave and pitch motions are almost out of phase for wave periods between 10 and 15 sec. Because the maximum wave elevation occurs near the northeast column and the vertical motion is very small, the minimum airgap occurs there. Moreover, a surrogate model based on radial basis function (RBF) has been built and adopted to estimate the hydrodynamic performance of TLP. A multi-objective evolutionary algorithm, Non-dominated Sorting Genetic Algorithm II (NSGAII), is employed to find the Pareto-optimal solutions. By comprehensive and systematic computations and analyses, it is revealed that the maximum dynamic tension shows positive correlation with pontoon height and width, but negative correlation with hull draft, column spacing, and column diameter. The most efficient modification strategy for design is proposed to reduce the maximum dynamic tendon tension. According to the strategy, the column spacing, draft, and column diameter should be increased in sequence. By applying this strategy, the maximum dynamic tendon tensions can be reduced while the total weight of the platform is minimized as much as possible.
\end{abstract}

\section{Introduction}

As the offshore oil/gas exploration moves toward deep water, floating production platforms, such as Floating Production Storage and Offloading (FPSO), Semi-submersible (SEMI), Tension Leg Platform (TLP), and Spar, have been widely adopted in recent years (API RP 2SK, 2005). Different from the other three floater concepts either using taut or semi-taut mooring systems, TLP is a compliant floating platform moored to seabed by several tendons which are pretensioned by excess buoyancy over total structural weight. The stability and vertical motion performance (heave, roll, and pitch) of TLP are much better than FPSO and traditional SEMI.

\footnotetext{
${ }^{*}$ Corresponding author; Director, Innovative Marine Hydrodynamics Laboratory (iMHL), Shanghai Jiao Tong University Email address: xinshuz@sjtu.edu.cn (Xinshu Zhang)
} 
Because of TLP's excellent motion performance, production wellheads and dry tree can be placed on the deck so that the cost for fabrication, production, and maintenance can be reduced accordingly. In conceptual or preliminary design phases, hull sizing is one of the most important task which can have significant effects on global motion performance and total cost for a project. In practice, hull sizing is an iterative process, which can be called as 'design spiral' (API RP 2T, 1997). It usually relies on designers' engineering experience acquired from the previous projects. Therefore, through conventional design approach, it's very difficult and time-consuming to achieve an optimal design for better global motion performance and lower cost.

A few researchers have put forward the optimization methods in order to achieve the optimal hydrodynamic performance for various offshore floating structures. For example, Akagi et al. (1984) applied generalized reduced gradient algorithm (GRGA) on hull-form optimization for a SEMI, in which the displacement, variable load, and heave motion were analytically formulated as objectives. Clauss and Birk (1996) used a commercial code, WAMIT, to evaluate the hydrodynamic performance of an arbitrary hull form, instead of using simplified formulae. In the study, nonlinear programming approach (NLP) was introduced to seek the minimum downtime of three different types of offshore structures, including gravity base structure (GBS), TLP, and SEMI. In order to reduce the design variables, Vannucci (1996) simplified the configuration of a TLP by a cylinder with two additional square plates. Based on this simplified model, the minimum weight was obtained by taking stability and hydrodynamic performance as constraints. Birk et al. (2004) compared the differences on improving the seakeeping performance between the deterministic method, such as Sequential Quadratic Programming (SQP), and the global methods, such as Genetic Algorithm (GA) and Adaptive Simulated Annealing (ASA). In their study, the hydrodynamic behavior of each design was estimated using WAMIT. Lee et al. (2007) utilized SQP and GA for the optimization of TLP by considering tendon fatigue life as an objective. Different from this previous hydrodynamic computations, where tendon tensions were simplified by equivalent linear spring, fully coupled analyses, comprised of frequency-domain and time-domain coupled analyses, were conducted by Lee and Lim (2008). More efficient hull shape optimization was obtained based on more accurate prediction of TLP's dynamic behaviors. Birk (2009) adopted a variant of multi-objective evolutionary algorithm ( $\varepsilon$-MOEA) for minimizing heave motion while maximizing deck load of a SEMI-type floater. They integrated parametric design tools, hydrodynamic analyses code, and multi-objective optimization algorithms to find Pareto-optimal solutions. To optimize the hull and mooring configuration for a SEMI, Williams (2012) took various conditions, such as quayside hull-topsides integration, wet tow, and in-place operating conditions, into consideration in stability and global performance analyses. Park et al. (2015) selected heave motion response and total steel weight as objectives to optimize a SEMI floating production unit (FPU). Simulated Annealing (SA) algorithm was employed with WADAM (DNV, 2013) for the hull form optimization. It was found that the column height was proportional to the total steel weight and inversely proportional to heave motion response. Kim and Jang (2016) extended the technique to optimize a TLP with tendon system. The results indicated that the pontoon volume and the sectional area of tendons are the dominant factors affecting motion performance and construction cost. Sugita and Suzuki (2016) developed a code for the optimization of a TLP for better global hydrodynamic performance, stability, and structural strength, based on Genetic Algorithm (GA) and Simulated Annealing (SA) algorithm, which proved to be efficient in seeking the optimal hull shape without using any 
empirical parameters. More recently, Zhang et al. (2017) performed extensive parametric study for a SEMI FPU, providing a benchmark for the future automated hull form optimization. They found that increasing draft could reduce the heave motion effectively, but increasing column width, pontoon width, or pontoon height would increase the heave motion.

Two essential problems should be considered properly in the optimization process for offshore floating structures. The first is on the selection of optimization algorithms. Based on previous studies, the global optimization algorithms, such as GA and SA, have shown their superiorities over the local optimization algorithms (i.e., GRG, NLP, and SQP). Meanwhile, the multi-objective optimization methods are required because there are always several objectives needed to be optimized simultaneously. Although the multi-objective global optimization algorithms require more computing time than the local methods, they attract more attention and have been widely applied in various fields in recent years. The second one is on the method to evaluate the hydrodynamic performance. When the computing technology is limited, the performance of floater is estimated by simplified models. With the rapid development in marine hydrodynamics, a few softwares, such as WAMIT and CHARM3D (Kim, 1997), prove to be efficient in computing the hydrodynamic coefficients and motion responses.

The present study is focused on applying a multi-objective evolutionary algorithm to optimize a TLP for better hydrodynamic performance and economy. A code called Innovative TLP Optimization Program (ITOP) has been developed. The multi-objective optimization scheme is implemented to find Pareto-optimal solutions instead of converting to a single-objective optimization problem. To raise the global optimization efficiency, a surrogate model based on radial basis function (RBF) has been built in order to compute the performance of each different hull configuration. A typical conventional TLP is selected for the present study. The base TLP is comprised of four circular columns, connected to a ring pontoon with a rectangular cross-section. Hull draft, column spacing, column diameter, pontoon height, and pontoon width are the selected five design variables to be optimized. The sample points used for constructing surrogate model are selected based on the initial design. DNV software WADAM (DNV, 2013; Payne et al., 2008; Matos et al., 2011), which has been extensively validated for the prediction of the wave-induced motion responses, is employed for hydrodynamic computations and analyses. As hull form is varied, the panel model and Morison's model are rebuilt and the motion responses are recomputed by WADAM and POSTRESP (DNV, 2007). The RBF-based surrogate model is employed to evaluate the hydrodynamic performance of the varied hull form during optimization. To minimize the most probable maximum (MPM) dynamic tendon tension and total structural weight, a multi-objective evolutionary algorithm, Non-dominated Sorting Genetic Algorithm II (NSGAII), is applied. Finally, Pareto-optimal solutions have been obtained and validated by direct numerical simulations.

\section{Mathematical Formulation}

In the phase of conceptual design, principal dimension of the hull and mooring and riser systems are preliminarily determined considering the environmental conditions and functional requirements. The conceptual selection results are taken as an initial design for further optimization in the present study. The key parameters of the selected initial design include design variables, which are varied frequently in order to achieve optimal 
values, and control variables, which are kept constant during optimization process. Five design variables include hull draft, column spacing, column diameter, pontoon height, and pontoon width. The other parameters such as topside weight, freeboard, and sectional area of tendons are fixed. The upper and lower bounds of each design variable are determined considering geometric and practical engineering feasibility. Based on design of experiment (DOE) theories (Anderson and Whitcomb, 1970), a set of sample points can be selected in the searching field. Every sample point represents a feasible design with corresponding hydrodynamic performance and weight. After computing hydrodynamic performance for every sample point, a surrogate model can be built based on the obtained numerical solutions. Therefore, the computing time for evaluating hydrodynamic performance of an arbitrary hull configuration decreases rapidly compared with direct numerical simulations. Multi-objective optimization algorithm is employed using the obtained surrogate model. The total structural weight and dynamic tendon tension are selected as objective functions with a number of constraints. Through a number of iterations, the optimal designs, called Pareto-optimal solutions, are obtained. The flowchart of the overall optimization process is illustrated in Figure 1.

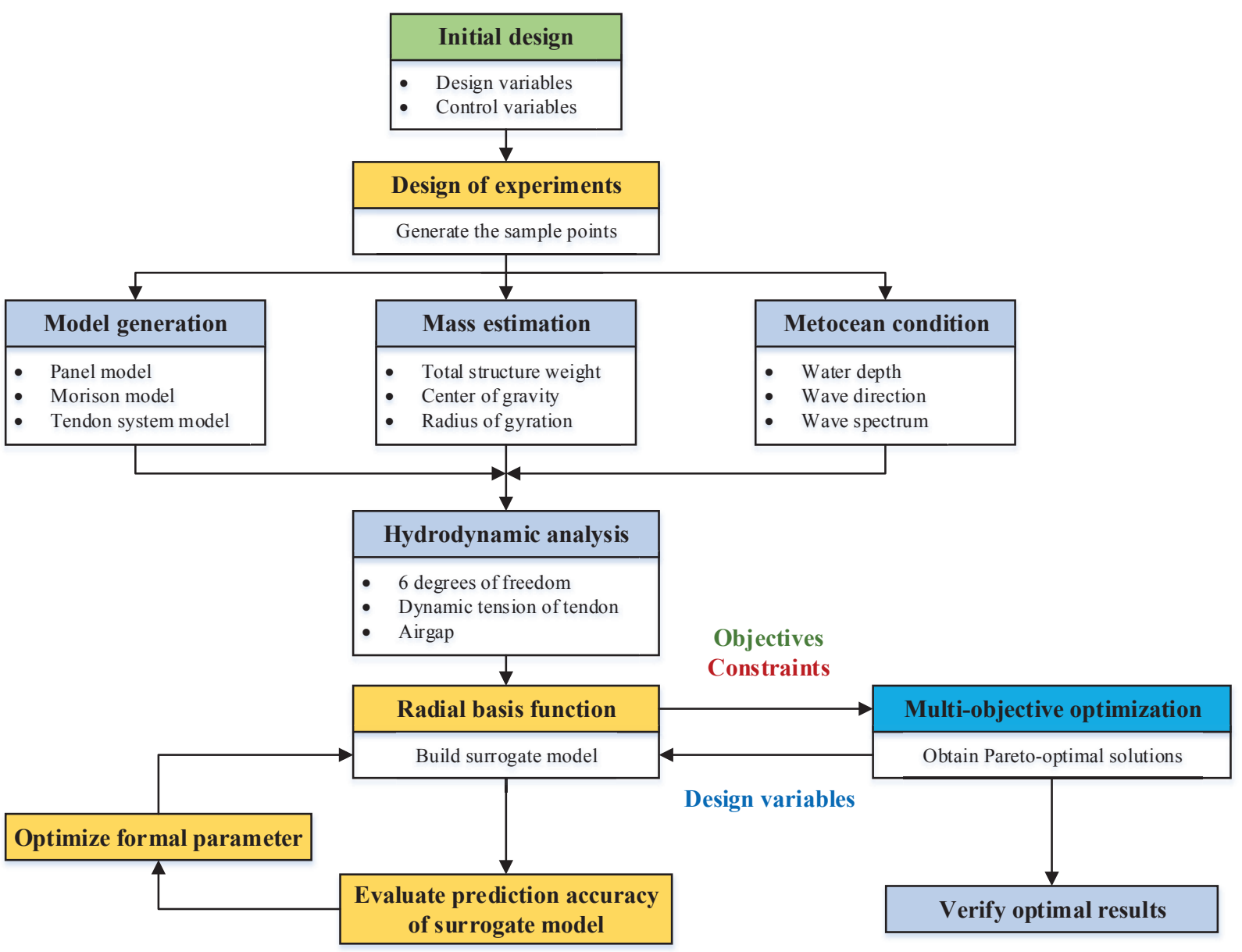

Figure 1: Flowchart of overall optimization process. Gray modules: numerical simulations for hydrodynamic estimation; Yellow modules: construction of surrogate models; Blue modules: iterative optimization process; Green module: an initial design obtained from conceptual design stage.

\subsection{Hydrodynamic analysis method}

The main dimensions of a base TLP are illustrated in Figure 2. Two coordinate systems are employed: the 


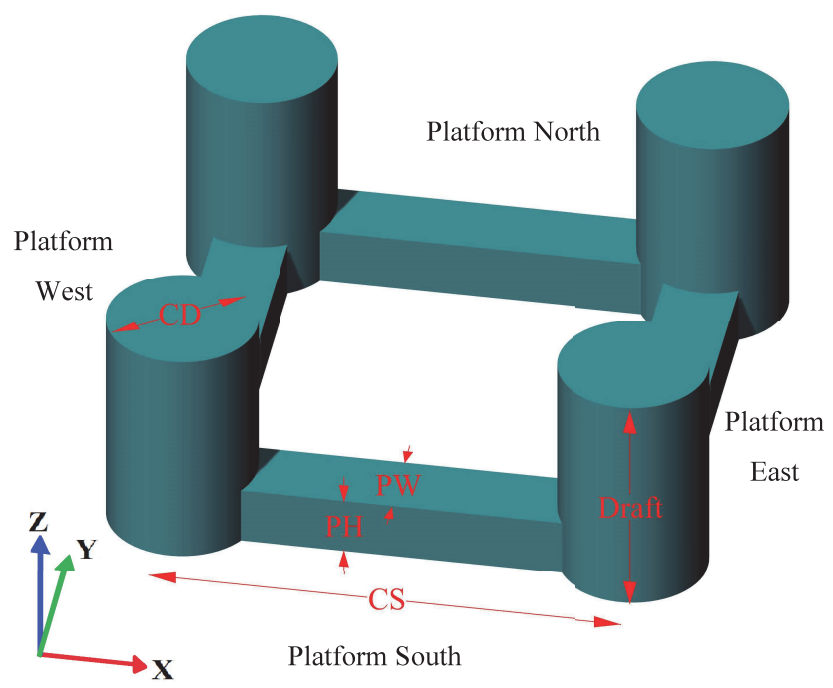

Figure 2: Definition of the main particulars of the base TLP. CD: column diameter; CS: column spacing; PW: pontoon width; PH: pontoon height.

$\boldsymbol{x}_{\mathbf{0}}$ system is fixed to the mean position of the floating body, and the $\boldsymbol{x}$ system is fixed to the moving hull. The boundary value problem is solved in the right-hand fixed coordinate system, as shown in Figure 2. The x-axis points to the direction of platform east $(\mathrm{PE})$ and the z-axis points upward. The origin is on the calm water plane at mid-section of the hull.

The definition of the incident wave heading is illustrated in Figure 3, where a plan view of the TLP is presented.

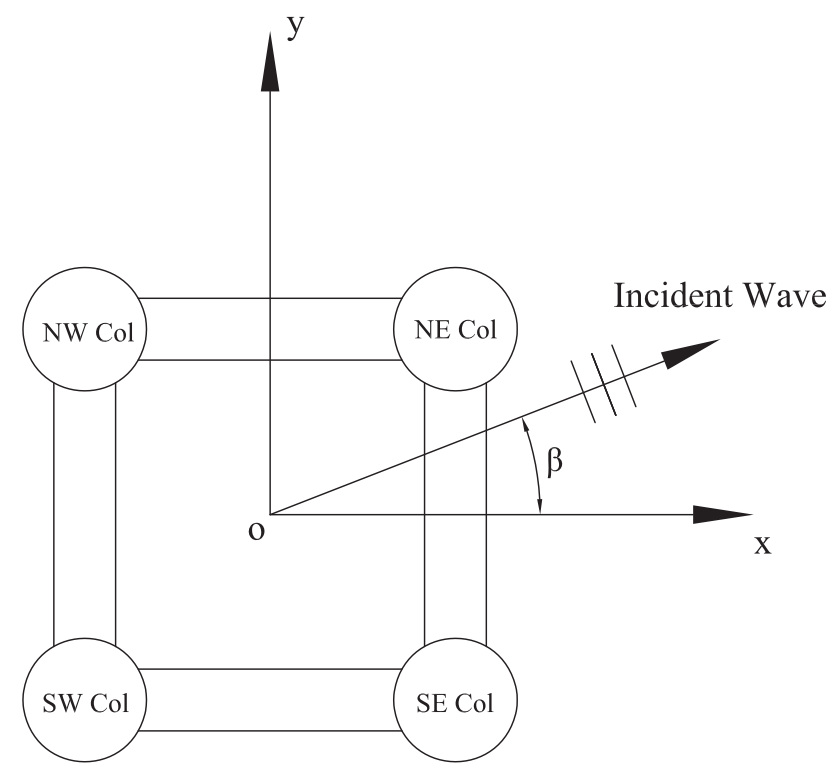

Figure 3: Definition of incident wave heading $\beta$ (Plan View) and index of four columns. NW Col: northwest column; NE Col: northeast column; SW Col: southwest column; SE Col: southeast column.

In the present study, hydrodynamic loads are computed based on potential flow theory. The loads induced by wind, current, and higher-order harmonic wave forces are neglected in the present optimization model. In 
order to compute the wave exciting force and drag force acting on wetted surface, panel method and Morison's equation are both adopted. After evaluating the wave-induced loads, the 6 degrees of freedom (DoF) equations of motion can be solved in frequency domain. The motion responses of the TLP in irregular waves are evaluated as the linear superposition of the responses in regular waves.

\subsubsection{Panel method}

In frame of potential flow theory, the fluid is assumed incompressible and the flow irrotational. Velocity potential function $\Phi(x, y, z, t)$ is introduced to describe the fluid motion. When the amplitude of incident wave is small and the body motion is stable, the component of time-dependent term is separated out as follow

$$
\Phi(x, y, z, t)=\operatorname{Re}\left[\phi(x, y, z) \mathrm{e}^{-i \omega t}\right]
$$

where $\phi(x, y, z)$ denotes the spatial velocity potential function and $\omega$ represents the wave angular frequency.

For computations of the first-order wave forces, the total velocity potential can be decomposed into different components as follow

$$
\Phi(x, y, z, t)=\Phi_{I}(x, y, z, t)+\Phi_{D}(x, y, z, t)+\Phi_{R}(x, y, z, t)
$$

where $\Phi_{I}, \Phi_{D}$, and $\Phi_{R}$ denote the velocity potentials induced by incident waves, diffracted waves, and radiated waves, respectively.

The wetted surface is discretized into a number of flat panels. The source strengths are assumed to be constant over each panel. The velocity potential in the fluid can be expressed as the superposition of the potentials induced by source points distributed on hull. Through solving discretized boundary integral equations, the source strengths and the velocity potential are obtained. Furthermore, the hydrodynamic pressures on the wetted surface are computed. The panel model for the base TLP is illustrated in Figure 4.

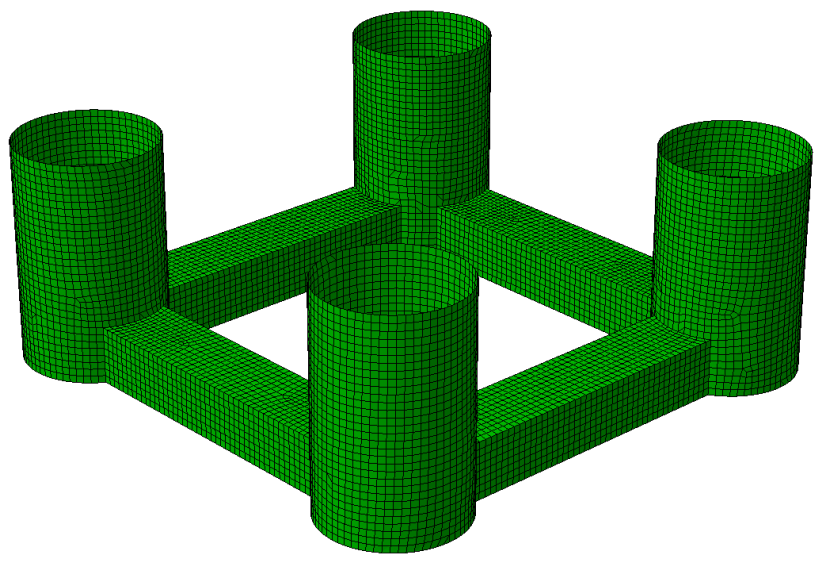

Figure 4: Panel model for the initial design. The hull of the TLP includes four columns $(\mathrm{draft}=31 \mathrm{~m}$, column spacing $=60 \mathrm{~m}$, column diameter $=20 \mathrm{~m}$ ) and four pontoons (pontoon height $=8 \mathrm{~m}$, pontoon width $=10 \mathrm{~m}$ ). Mesh density $=2.5 \mathrm{~m}$; number of panels $N=2864$.

\subsubsection{Morison's model}

Morison's equation is an empirical formula for evaluating the force acting on the element based on the assumption that the wave system does not have diffraction and radiation due to the presence of Morison 
element. Morison's equation is written as

$$
F=\rho V_{M}\left(1+C_{a}\right) \dot{v}+\frac{1}{2} \rho \sigma C_{D} v|v|
$$

where the first term and second term on the right side are the inertia force and drag force, respectively; $\rho$ is density of the fluid; $V_{M}$ is displaced volume of an element; $v$ and $\dot{v}$ are the horizontal undisturbed fluid velocity and acceleration, respectively; $\sigma$ is the projected area of the element; The added mass $C_{a}$ and drag coefficients $C_{D}$ are determined based on the shape of the element. In the present study, inertia force is computed using panel method, and the drag force is evaluated by Morison's equation. The Morison's model for the base TLP is illustrated in Figure 5.

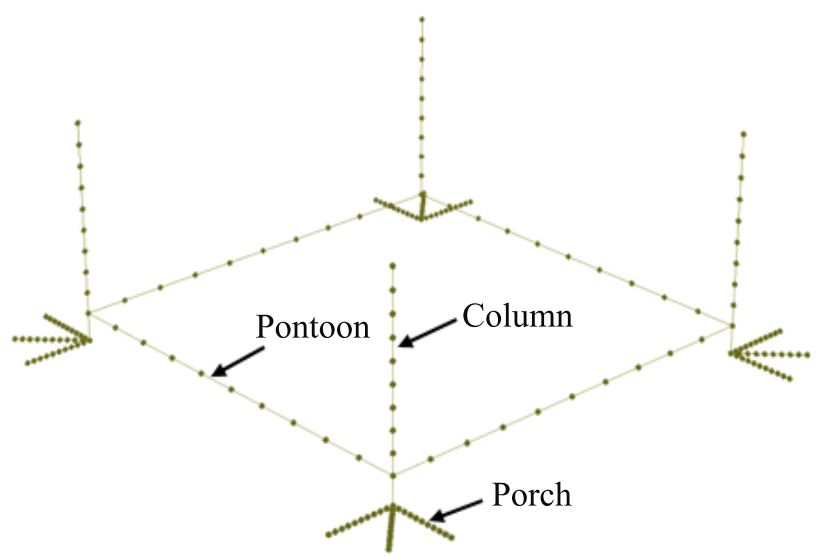

Figure 5: Morison model for the initial design. Number of Morison elements is $\mathrm{N}=200$.

\subsubsection{Equations of motion}

By adopting panel method and Morison's equation, the hydrodynamic loads acting on the floater are computed. The equations of motion are solved in frequency domain to predict the motion responses in regular waves. The equations of motion are written as

$$
\left[-\omega^{2}(\mathbf{M}+\mathbf{A}(\omega))+i \omega\left(\mathbf{B}_{p}(\omega)+\mathbf{B}_{v}\right)+\mathbf{C}+\mathbf{C}_{e}\right] \boldsymbol{\xi}(\omega, \beta)=\mathbf{F}(\omega, \beta)
$$

where $\boldsymbol{\xi}(\omega, \beta)=\left(\xi_{1}, \xi_{2}, \xi_{3}, \xi_{4}, \xi_{5}, \xi_{6}\right)$ denotes the displacement vector of rigid body for wave frequency $\omega$ and incident wave heading $\beta ; \mathbf{A}(\omega)$ and $\mathbf{B}_{p}(\omega)$ are the added mass and radiation damping matrix, respectively; $\mathbf{M}$ represents the mass/inertia matrix; $\mathbf{B}_{v}$ denotes the linearized viscous damping matrix; $\mathbf{C}$ is the hydrostatic restoring matrix; $\mathbf{C}_{e}$ is the external restoring matrix induced by the tendons or mooring system; $\mathbf{F}(\omega, \beta)$ denotes the first-order wave exciting force.

In the equations of motion, the frequency-dependent $\mathbf{A}, \mathbf{B}_{p}, \mathbf{B}_{v}$, and $\mathbf{F}$ are obtained by panel method and Morison's equation. The accurate estimation of mass is a very complicated task because the steel weight of the platform, equipment, and variable loads are difficult to estimate at early design stage. It is assumed that the steel weight is proportional to the volume of the platform. The proportionality coefficients for different components of the floater are listed in Table 1. In addition, a 15\% margin is added to account for the outfitting weight. The topside weight is fixed as a constant based on functional requirements. 
Table 1: Proportionality coefficients for mass estimation.

\begin{tabular}{lcc}
\hline Component & Unit & Coefficient \\
\hline Column & ton $/ \mathrm{m}^{3}$ & 0.16 \\
Node & ton $/ \mathrm{m}^{3}$ & 0.19 \\
Pontoon & ton $/ \mathrm{m}^{3}$ & 0.24 \\
\hline
\end{tabular}

The hydrostatics restoring matrix is computed by

$$
C=\left[\begin{array}{cccccc}
0 & 0 & 0 & 0 & 0 & 0 \\
0 & 0 & 0 & 0 & 0 & 0 \\
0 & 0 & \rho g S & \rho g S_{y} & -\rho g S_{x} & 0 \\
0 & 0 & \rho g S_{y} & \rho g\left(S_{y y}+V_{w} z_{B}\right)-m g z_{G} & -\rho g S_{x y} & -\rho g V_{w} x_{B}+m g x_{G} \\
0 & 0 & -\rho g S_{x} & -\rho g S_{x y} & \rho g\left(S_{x x}+V_{w} z_{B}\right)-m g z_{G} & -\rho g V_{w} y_{B}+m g y_{G} \\
0 & 0 & 0 & -\rho g V_{w} x_{B}+m g x_{G} & -\rho g V_{w} y_{B}+m g y_{G} & 0
\end{array}\right]
$$

where $\left(x_{G}, y_{G}, z_{G}\right)$ is the center of gravity, $\left(x_{B}, y_{B}, z_{B}\right)$ the center of buoyancy, $\rho$ the density of the fluid, $g$ the acceleration of gravity, $V_{w}$ the displacement, $S$ the waterplane area; $S_{x}$ and $S_{y}$ are the first-order moments of waterplane area to $x$ and $y$ axes, respectively; $S_{x x}$ and $S_{y y}$ are the second-order moments of waterplane area to $x$ and $y$ axes, respectively.

The mooring elements of TLP are tendons connecting the platform to the seabed. In the present study, the tendons are simplified by linear springs, because the motion of platform is small comparing to the length of tendons. The external restoring matrix due to the stiffness of the tendons varies as the platform moves in horizontal direction. The external restoring matrix induced by tendons is formulated as

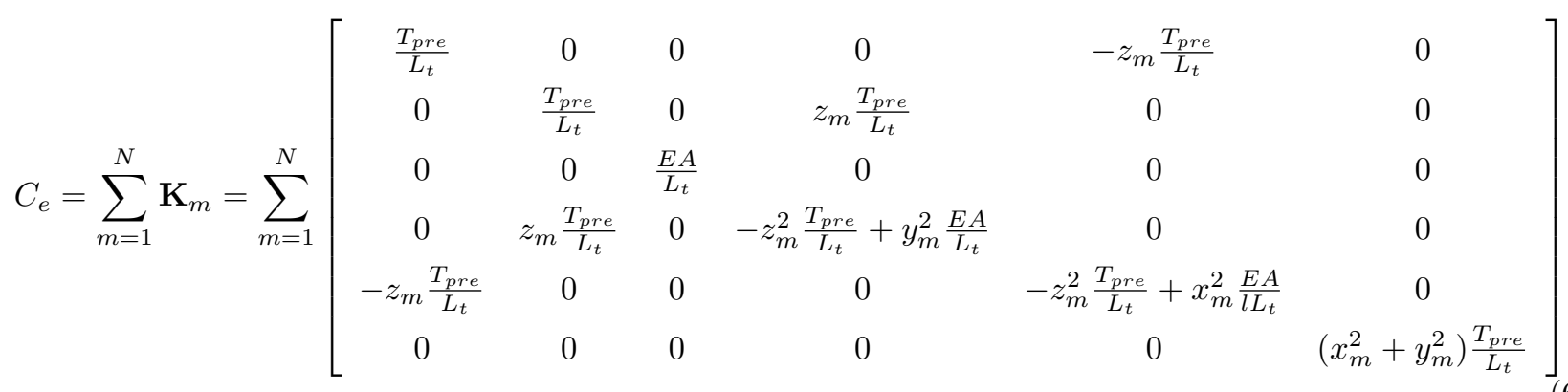

where $N$ is the number of tendons, $E$ the elasticity modulus of the material of tendon, $A$ the cross-section area of each tendon, $L_{t}$ the length of the tendon measured from seabed to porch, $\left(x_{m}, y_{m}, z_{m}\right)$ the coordinate of the TLP tendon tip, $T_{\text {pre }}$ the pretension of each tendon induced by the exceeding buoyancy over the weight, which can be computed by

$$
T_{\text {pre }}=\frac{\triangle-W}{N}
$$

where $\triangle$ denotes the displacement of the platform; $W$ is the total weight of the platform.

Upon solving the equations of motion, the dynamic tendon tension can be evaluated based on the assumption that the mooring is equivalent to a linear spring. By solving the equations of motion in frequency domain, the motion responses in regular waves can be obtained. In order to compute the motion responses in irregular 
waves, a wave spectrum denoted by $S_{w}$ should be used. In the present study, JONSWAP wave spectrum is employed. The response spectrum $S_{r}$ can be computed by

$$
S_{r}(\omega, \beta)=\int_{-\pi / 2}^{\pi / 2}|H(\omega, \epsilon)|^{2} S_{w}(\omega) f(\alpha) d \alpha
$$

where $\beta$ is the wave heading (main direction of the irregular wave system); $H$ denotes the response amplitude operator $(\mathrm{RAO})$ in $6 \mathrm{DoF} ; \epsilon=\beta+\alpha$ represents the heading angle between the elementary sinusoidal waves and the $\mathrm{x}$-axis; $\alpha$ is the angle of the elementary waves relative to the main direction of the irregular wave system; $f(\alpha)$ is a wave spreading function only for short-crested waves, representing the directional distribution of energy in the waves. According to Rayleigh distribution, the most probable maximum (MPM) response in short-term sea state, such as 3-hour storm, can be computed by

$$
A_{\max }=\sqrt{\left(2 m_{0} \log \frac{t}{T_{2}}\right)}
$$

where $A_{\max }$ represents the MPM response in $t ; m_{0}$ denotes the zero moment of the response spectrum; $t$ is the time duration (3 hours adopted in the present paper) and $T_{2}$ is the zero-upcrossing period of the response spectrum.

\subsubsection{Airgap computations}

The airgap is defined as the minimum distance measured from wave crest to the bottom of deck. It is one of the most important design criteria for evaluating the wave slamming events. For airgap, the setdown of the platform due to the offset should be taken into account. The airgap can be formulated as

$$
A G=A G_{\text {static }}-Z_{\text {elevation }}-Z_{\text {setdown }}+\left(\xi_{3}-\xi_{5} x+\xi_{4} y\right)
$$

where $A G$ represents the airgap at a specified location; $A G_{\text {static }}$ is the static airgap in calm water, which is equivalent to the freeboard. In the present study, the freeboard is fixed to $25 \mathrm{~m}$. $Z_{\text {setdown }}$ denotes a steady displacement in vertical direction. When the platform has an offset induced by wind, wave, and current loads, the draft will increase because of the restraint of tendons. The increased draft is called 'setdown'. $Z_{\text {elevation }}$ represents the absolute wave elevation due to incident wave, diffracted wave, and radiated wave. $(x, y)$ denotes the horizontal coordinate of a specified point. The term $\left(\xi_{3}-\xi_{5} x+\xi_{4} y\right)$ is the vertical combined motion of a point on still water surface.

\subsection{Surrogate model}

Surrogate model is efficient for rapid optimization analyses (Audet et al., 2000). The application of surrogate model can improve the computing efficiency significantly while achieving accurate prediction. Design of experiments, related with the strategy of assigning sample points, is one of the important tasks which should be considered during the construction process of surrogate model. At present, the developed design of experiments mainly fall into two categories: space-boundary-based design and space-filling-based design (Forrester and Keane, 2009). The design of experiments based on space filling, including full factorial design, orthogonal design, uniform design, and latin hypercube design, becomes more feasible when the dimension of design variables increases. In addition, the mathematical relationship between input and output is also important 
to achieve high efficiency and accuracy. The commonly used surrogate models include response surface (RS) model, Kriging model, radial basis function (RBF), and support vector machine (SVM). In the present study, the radial basis function (RBF) is adopted to construct the surrogate model since RBF model is very convenient to find the optimal form parameter and good at function fitting.

\subsubsection{Design of experiments}

Full factorial design strategy is adopted to select sample points. In theory of DOE, design variables are called 'factors', each with several different values, also called 'levels'. The full factorial design is an experiment consisting all possible combinations of these levels across all factors. The upper bound, lower bound, and level step of all factors are listed in Table 2. The level step is computed as (Upper bound - Lower bound)/(level number - 1).

Table 2: Full factorial design.

\begin{tabular}{ccccc}
\hline Factors & Upper bound & Lower bound & Level number & Level step \\
\hline Draft & $34.1 \mathrm{~m}$ & $27.9 \mathrm{~m}$ & 5 & $1.55 \mathrm{~m}$ \\
Column diameter & $22.0 \mathrm{~m}$ & $18.0 \mathrm{~m}$ & 3 & $2.00 \mathrm{~m}$ \\
Column spacing & $66.0 \mathrm{~m}$ & $54.0 \mathrm{~m}$ & 5 & $3.00 \mathrm{~m}$ \\
Pontoon width & $11.0 \mathrm{~m}$ & $9.0 \mathrm{~m}$ & 3 & $1.00 \mathrm{~m}$ \\
Pontoon height & $8.8 \mathrm{~m}$ & $7.2 \mathrm{~m}$ & 3 & $0.80 \mathrm{~m}$ \\
\hline
\end{tabular}

According to the permutation and combination theory, totally $5^{2} \times 3^{3}=675$ sample points are selected for training the surrogate model. Each sample point represents a specific hull configuration and the associated tendon tension and structural weight are selected as objectives. The surrogate model is adopted to build the correlation between input (platform dimension) and output (hydrodynamic performance).

\subsubsection{Radial basis function model}

The radial basis function (RBF) model is widely used in the field of data fitting. A general discussion of RBF is presented by Buhmann (2009). The RBF is also applied on seakeeping problem by Zhang et al. (2010). The sketch of radial basis function is illustrated in Figure 6 .

The radial basis function is a one-dimensional nonlinear function of Euclidean distance between the interpolation point and the sample points.

$$
g_{j}(\boldsymbol{x})=g\left(\left\|\boldsymbol{x}-\boldsymbol{x}_{j}\right\|\right), \quad j=1, \ldots, Q
$$

where $\boldsymbol{x}$ is the vector of interpolation point; $\boldsymbol{x}_{j}$ is the vector of the $j$ th sample point; $Q$ denotes the number of sample points. The approximating function $\boldsymbol{y}_{i}$ is represented as a sum of $Q$ radial basis functions $g_{j}\left(\boldsymbol{x}_{i}\right)$, each associated with a different center $\boldsymbol{x}_{j}$, and weighted by an appropriate coefficient $\boldsymbol{w}_{j}$.

$$
\begin{gathered}
\boldsymbol{y}_{i}=\sum_{j=1}^{Q} \boldsymbol{w}_{j} g_{j}\left(\boldsymbol{x}_{i}\right) \\
\boldsymbol{x}_{i}=\left[\begin{array}{llll}
x_{1 i} & x_{2 i} & \cdots & x_{M i}
\end{array}\right]^{T}
\end{gathered}
$$




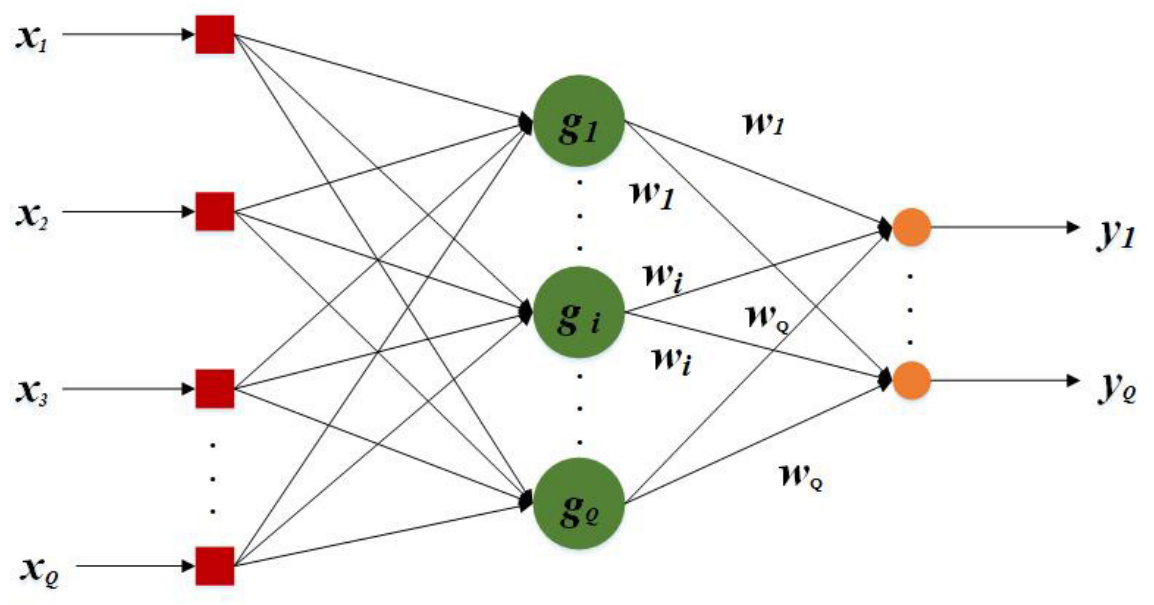

Figure 6: Sketch of RBF model. Design variables: $\left[\boldsymbol{x}_{1}, \boldsymbol{x}_{2}, \ldots, \boldsymbol{x}_{Q}\right]$; objective values: $\left[\boldsymbol{y}_{1}, \ldots, \boldsymbol{y}_{Q}\right]$; radial basis functions: $\left[\boldsymbol{g}_{1}, \ldots\right.$, $\left.\boldsymbol{g}_{i}, \ldots, \boldsymbol{g}_{Q}\right]$; weight: $\left[\boldsymbol{w}_{1}, \ldots, \boldsymbol{w}_{i}, \ldots, \boldsymbol{w}_{Q}\right]$. Square nodes: input layer; big circle nodes: hidden layer; small circle nodes: output layer.

$$
\boldsymbol{y}_{i}=\left[\begin{array}{llll}
y_{1 i} & y_{2 i} & \cdots & y_{N i}
\end{array}\right]^{T}
$$

where weights $\boldsymbol{w}_{j}$ and approximating function $\boldsymbol{y}_{i}$ share the same dimension. $M$ denotes the dimension of design variable. $N$ represents the dimension of objective value. It is assumed that the input matrix is $X$ and its corresponding output matrix is $Y$. They can be written as

$$
\begin{aligned}
X=\left[\begin{array}{llll}
\boldsymbol{x}_{1} & \boldsymbol{x}_{2} & \cdots & \boldsymbol{x}_{Q}
\end{array}\right]=\left[\begin{array}{cccc}
x_{11} & x_{12} & \cdots & x_{1 Q} \\
x_{21} & x_{22} & \cdots & x_{2 Q} \\
\vdots & \vdots & \ddots & \vdots \\
x_{M 1} & x_{M 2} & \cdots & x_{M Q}
\end{array}\right] \\
Y=\left[\begin{array}{lllll}
\boldsymbol{y}_{1} & \boldsymbol{y}_{2} & \cdots & \boldsymbol{y}_{Q}
\end{array}\right]=\left[\begin{array}{cccc}
y_{11} & y_{12} & \cdots & y_{1 Q} \\
y_{21} & y_{22} & \cdots & y_{2 Q} \\
\vdots & \vdots & \ddots & \vdots \\
y_{N 1} & y_{N 2} & \cdots & y_{N Q}
\end{array}\right]
\end{aligned}
$$

where $x_{i j}$ is the $i$ th design variable of the $j$ th sample point; $y_{i j}$ represents the $i$ th objective value of the $j$ th sample point.

As one of the commonly used radial basis function, Multi-Quadric function (MQ) is employed in the present study and can be written as

$$
g_{j}(\boldsymbol{x})=\sqrt{\left\|\boldsymbol{x}-\boldsymbol{x}_{j}\right\|^{2}+b^{2}}
$$

The value of formal parameter $b$ has a significant impact on the accuracy of the surrogate model and should be chosen properly. The accuracy of predicted outputs were tested by the leave-one-out cross validation (LOOCV) to find the best formal parameters. 
The radial basis functions $G$ can be obtained using the input matrix $X$ and can be written as

$$
G=\left[\begin{array}{cccc}
g_{1}\left(\boldsymbol{x}_{1}\right) & g_{1}\left(\boldsymbol{x}_{2}\right) & \cdots & g_{1}\left(\boldsymbol{x}_{Q}\right) \\
g_{2}\left(\boldsymbol{x}_{1}\right) & g_{2}\left(\boldsymbol{x}_{2}\right) & \cdots & g_{2}\left(\boldsymbol{x}_{Q}\right) \\
\vdots & \vdots & \ddots & \vdots \\
g_{Q}\left(\boldsymbol{x}_{1}\right) & g_{q}\left(\boldsymbol{x}_{2}\right) & \cdots & g_{Q}\left(\boldsymbol{x}_{Q}\right)
\end{array}\right] \in \mathbf{R}^{Q \times Q}
$$

The system (12) can be written in matrix form

$$
Y=W G
$$

The weights can be found after matrix inversion

$$
W=Y G^{-1}
$$

with

$$
W=\left[\begin{array}{llll}
\boldsymbol{w}_{1} & \boldsymbol{w}_{2} & \cdots & \boldsymbol{w}_{Q}
\end{array}\right]=\left[\begin{array}{cccc}
w_{11} & w_{12} & \cdots & w_{1 Q} \\
w_{21} & w_{22} & \cdots & w_{2 Q} \\
\vdots & \vdots & \ddots & \vdots \\
w_{N 1} & w_{N 2} & \cdots & w_{N Q}
\end{array}\right] \in \mathbf{R}^{N \times Q}
$$

\subsection{Multi-objective genetic algorithm}

Nowadays, most of the engineering problems involve a number of objectives to be optimized simultaneously. The challenge of these problems is that the objectives can conflict with each other, so the results of multiobjective optimization problems are a set of trade-off optimal solutions, also known as Pareto-optimal solutions. The genetic algorithm is one of the intelligent evolutionary algorithms, which can search for the global optimal points with random starting points. In the optimization process, the design variables are combined as an array, called 'individual'. Many different individuals form a population, which will vary with the operations of selection, crossover, and mutation in each generation. When the number of generation becomes large enough, the individuals are nearly the optimal solutions because of the natural rules of 'Natural selection and survival of the fittest' (Sivaraj and Ravichandran, 2011; Deb, 2001).

\subsubsection{Non-dominated sorting genetic algorithm II}

The Non-dominated Sorting Genetic Algorithm II (NSGAII) is one of the popularly used multi-optimization algorithms (Deb, 2011). The flowchart of the algorithm is illustrated in Figure 7.

Different from the traditional genetic algorithms, NSGAII introduces the concepts of non-dominated sorting and crowing distance in the genetic algorithm in order to realize the sequence of all individuals. If one individual $p$ is better than the other individual $q$ in terms of all objectives, then $p$ is defined as the domination of $q$. Otherwise, $p$ is non-dominated to $q$. In NSGAII algorithm, the non-dominated individuals share the same ranking value, while the higher ranking individuals dominate the lower ranking individuals. When the optimization process finishes, the highest ranking individuals are nearly the Pareto-optimal solutions. To obtain uniform distribution optimal solutions, the individuals with the same ranking value should be further ranked by the crowing distance, Euclidean distance between other individuals. Population trimming procedure can keep the population size as a fixed number. Through a number of iterations, the optimization can yield Pareto-optimal solutions. 


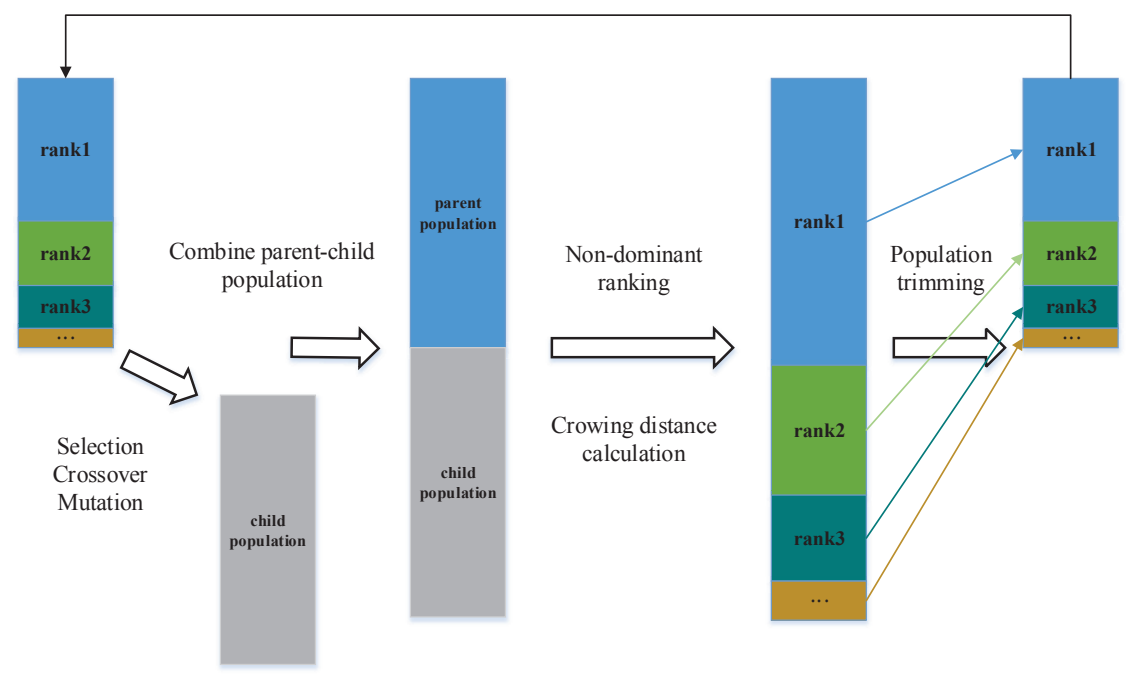

Figure 7: The description of the principle for non-dominated sorting genetic algorithm II (NSGAII).

\subsubsection{Penalty function}

The problems solved by NSGAII are usually unconstrained or linear constrained, but the constraints of the present study such as heave, surge, and airgap are nonlinear. A penalty function is introduced for the transformation from nonlinear constrained problems to unconstrained problems. The multi-objective optimization problem, in its general form, can be written as

$$
\begin{aligned}
\min & f(\mathbf{x})=\left[f_{1}, f_{2}, \ldots, f_{m}\right] \\
\mathbf{x} & =\left[x_{1}, x_{2}, \ldots, x_{n}\right] \\
\text { s.t. } & g_{i}(\mathbf{x}) \geqslant 0, \quad i=1, \ldots, k \\
& h_{j}(\mathbf{x})=0, \quad j=1, \ldots, l
\end{aligned}
$$

In the above mentioned problem, there are $m$ objective functions, $n$ variables, $k$ greater-than-equal-to type inequality constraints, and $l$ equality constraints. The function $f$ is the $\mathrm{m}$-dimensional multi-objective function. The design variables $\mathbf{x}$ are combined as an n-dimensional array. $g_{i}$ represents the $i$ th constraint which is a linear or nonlinear function. $h_{i}$ represents the $i$ th equation constraint. Penalty function can be written as

$$
P(\mathbf{x})=\sum_{i=1}^{k} \max \left[0,-g_{i}(\mathbf{x})\right]^{2}+\sum_{j=1}^{l}\left|h_{j}(\mathbf{x})\right|^{2}
$$

When $\mathbf{x}$ satisfies all of the constraints, the value of the penalty function is equal to zero, otherwise the penalty function is a positive number. By means of penalty function, the constrained optimization problem transforms the objectives as

$$
\min \quad F(\mathbf{x}, \sigma)=f(\mathbf{x})+\sigma P(\mathbf{x})
$$

where $\sigma$ is penalty factor, which is defined as a very large positive number. In the optimization procedure, the second term on the right side of Eqn.(27) will approach to zero, which means all of the constraints have been fulfilled. 


\section{Hydrodynamic Performance Analysis}

\subsection{Convergence tests}

To determine a proper mesh density for accuracy and efficiency consideration, the convergence tests are carried out for an initial design in $0^{\circ}$ wave heading. The main particulars and other parameters of the initial design are listed in Table 3.

Table 3: Main particulars of the initial design.

\begin{tabular}{lccc}
\multicolumn{4}{c}{ Table 3: Main particulars of the initial design. } \\
\hline Parameter & Symbol & Unit & Value \\
\hline Draft & Draft & $\mathrm{m}$ & 31 \\
Column diameter & $C D$ & $\mathrm{~m}$ & 20 \\
Column spacing & $C S$ & $\mathrm{~m}$ & 60 \\
Pontoon width & $P W$ & $\mathrm{~m}$ & 10 \\
Pontoon height & $P H$ & $\mathrm{~m}$ & 8 \\
Freeboard & $F B$ & $\mathrm{~m}$ & 25 \\
Deck height & $D H$ & $\mathrm{~m}$ & 15 \\
\hline Deck steel weight & $W_{\text {deck }}$ & ton & 8000 \\
Equipment weight & $W_{\text {equip }}$ & ton & 12000 \\
Hull steel weight & $W_{h u l l}$ & ton & 17900 \\
Vertical center of gravity & $V C G$ & $\mathrm{~m}$ & 12.1 \\
Radius of gyration & $\left(R_{x x}, R_{y y}, R_{z z}\right)$ & $\mathrm{m}$ & $(31,31,40)$ \\
\hline Pretension of tendon & $T_{p r e}$ & ton & 1285 \\
Outer diameter of tendon & $O D$ & $\mathrm{~cm}$ & 71.1 \\
Inner diameter of tendon & $I D$ & $\mathrm{~cm}$ & 65.0 \\
Length of tendon & $L_{t}$ & $\mathrm{~m}$ & 969 \\
Stiffness of tendon & $K_{e}$ & $\mathrm{~N} / \mathrm{m}$ & $1.36 \times 10^{7}$ \\
\hline
\end{tabular}

Four different sets of panelization are tested. Because of the symmetry, only a quarter of the model is built and meshed in order to speed up the computations. As shown in Figure 8, the hydrodynamic behaviors converge as the number of panel increases. Based on the convergence tests, the mesh size is set to $2.5 \mathrm{~m}$ in the following hydrodynamic performance analysis and overall optimization process. As shown in Figure 8(a), the non-dimensional heave added mass coefficient $\mu_{33}$ varies between 0.6 and 0.7 as the wave period varies from 3 sec to 30 sec. Meanwhile, by using 4000 panels, the maximum added mass is approximately 0.62 around 12 sec. The non-dimensional radiation damping coefficient $\lambda_{33}$, plotted in Figure $8(b)$, drops to a small number as wave period goes to around $7.5 \mathrm{sec}$ and $14 \mathrm{sec}$. The non-dimensional first-order wave exciting force in heave reaches the minimum around the wave period of $6.5 \mathrm{sec}$, which is caused by the cancellation effect between pontoons. As shown in Figure $8(c)$ and $(d)$, the local hump in heave RAO is around 4 sec, while no hump can be observed in heave exciting force. This is due to the natural period of the TLP in heave. It is about 4 sec, computed by

$$
T_{n}=2 \pi \sqrt{\frac{M+A_{33}}{K}}
$$


where $K=12 K_{e}$ is the stiffness in heave. Large motion amplitude can be induced by the small wave load in resonant condition.

\subsection{RAOs in regular waves}

The response amplitude operators (RAOs) of $6 \mathrm{DoF}$ are essential for further motion predictions in irregular waves. Totally four different wave headings, $0^{\circ}, 15^{\circ}, 30^{\circ}$, and $45^{\circ}$, are computed with consideration of the symmetric hull configuration. The numerical results are presented in Figure 9. As shown, the heave RAOs are relatively small comparing with the other two translational motion modes. In addition, the roll and pitch RAOs are smaller than yaw RAOs. It confirms that the TLP is stiff out of the waterplane (heave, roll, and pitch) and flexible within the waterplane (sway, surge, and yaw). As illustrated in Figure 9(c), the heave RAOs are not sensitive to wave headings. The heave RAOs converge to a constant value when the incident wave period goes to infinity. The convergency value can be solved by static equilibrium equation, which can be written as

$$
\lim _{T \rightarrow \infty} \mathrm{RAO}_{\text {heave }}=\frac{\rho g A_{w}}{\rho g A_{w}+K_{e}}
$$

where $T$ denotes the period of regular wave, $\rho$ the density of water, $g$ the acceleration of gravity, $A_{w}$ the waterplane area of the platform, $K_{e}$ the stiffness induced by the total tendons. In the present study, the theoretical solution of heave RAOs at zero frequency is 0.057 , which is consistent with the variation tendency obtained by numerical computations, as shown in Figure 9(c).

It should be noted that the tendon tension RAOs are important design criteria for the TLP. As shown in Figure 10, the tendons are labeled. No.2, No.5, No.8, and No.11 tendons are selected for the following analyses. The corresponding tendon tension RAOs are illustrated in Figure 11. As shown, the wave heading can affect the tendon tension RAOs significantly when the incident wave period is less than 20 sec. As the wave period increases, all tendon tension RAOs converge to a same constant value.

\subsection{Maximum response evaluation in irregular waves}

In the present study, the MPM responses are computed based on Rayleigh distribution. 100-year hurricane condition in west central Gulf of Mexico is adopted (API RP 2MET, 2014) as the survival condition. JONSWAP wave spectrum is applied and the wave parameters are listed in Table 4.

Table 4: Wave parameters of JONSWAP spectrum for survival condition.

\begin{tabular}{cccccc}
$H_{s}(\mathrm{~m})$ & $T_{p}(\mathrm{sec})$ & $T_{z}(\mathrm{sec})$ & $\gamma$ & $\sigma_{a}$ & $\sigma_{b}$ \\
\hline 12.3 & 14.4 & 11.17 & 3.3 & 0.07 & 0.09 \\
\hline
\end{tabular}

The computed response spectra in long-crest waves are plotted in Figure 12. As shown in Figure 12(a), the heave motion response spectra are almost the same for different wave directions. As shown in Figure 12(b), the peak periods of the surge motion response spectra are also the same (near $15 \mathrm{sec}$ ) for different wave headings. Figure 12(c) $(f)$ illustrate the comparison of tendon tension for four different wave headings. The responses of No.5 and No.8 tendons are more significant than the other two tendons. This is due to the fact that heave and pitch motions are almost out of phase (phase shift is around $180^{\circ}$ ) for wave periods between 10 and $15 \mathrm{sec}$, so that the vertical motions at No.5 and No.8 tendon tips are larger than those for No.2 and No.11 tendons. 
(a)

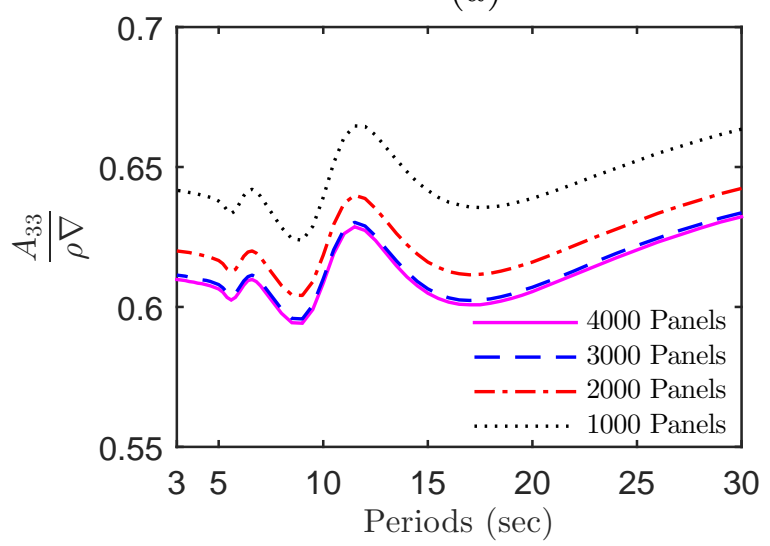

(c)

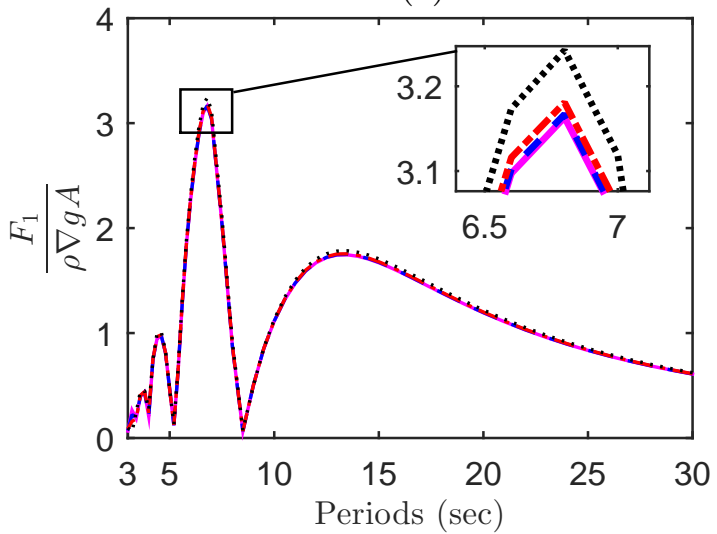

(e)

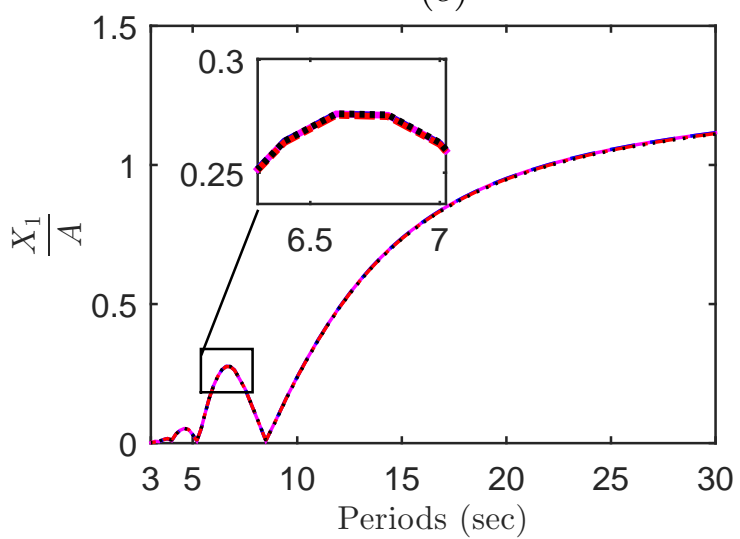

(b)

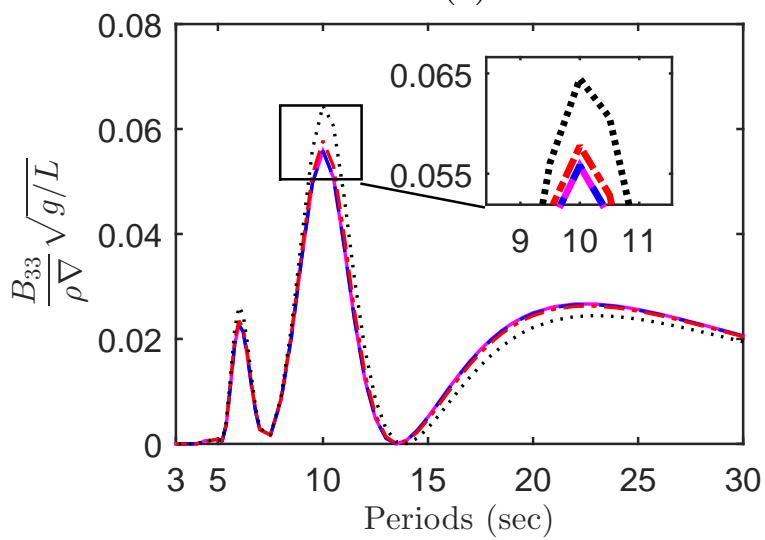

(d)

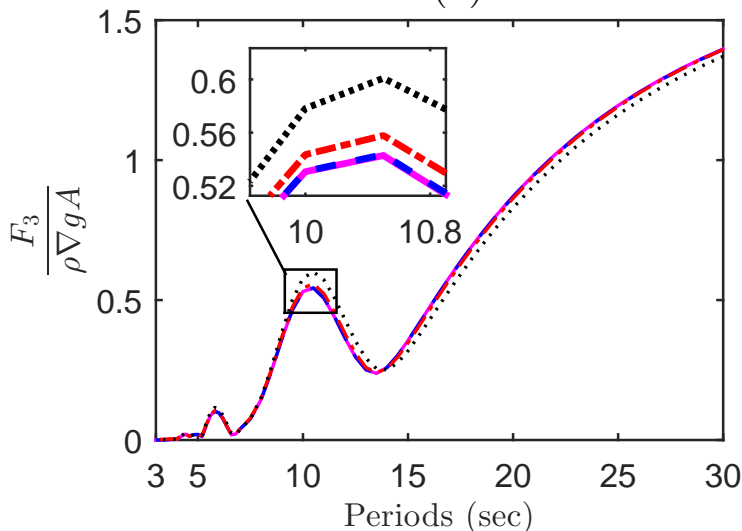

$(f)$

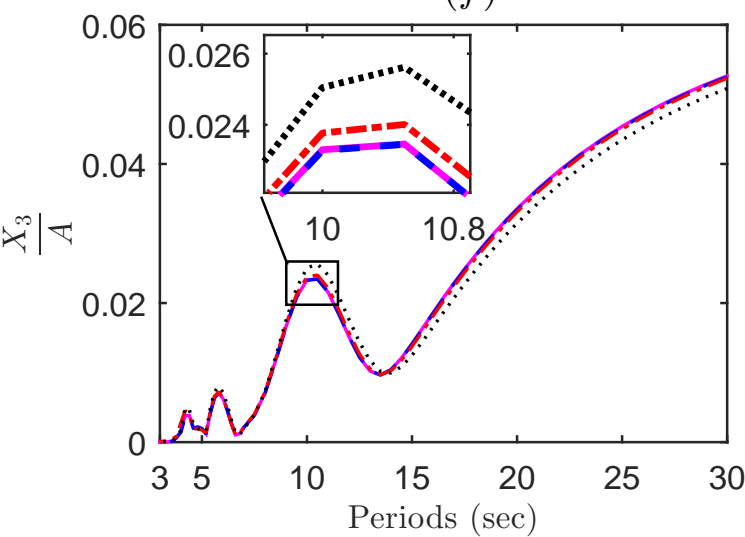

Figure 8: Convergence test for the base TLP. (a) Non-dimensional added mass in heave; (b) Non-dimensional radiation damping in heave; $(c)$ Non-dimensional first-order wave exciting force in surge; $(d)$ Non-dimensional first-order wave exciting force in heave; (e) Surge RAOs; $(f)$ Heave RAOs. A is the amplitude of incident wave; L is the characteristic length of the TLP. 

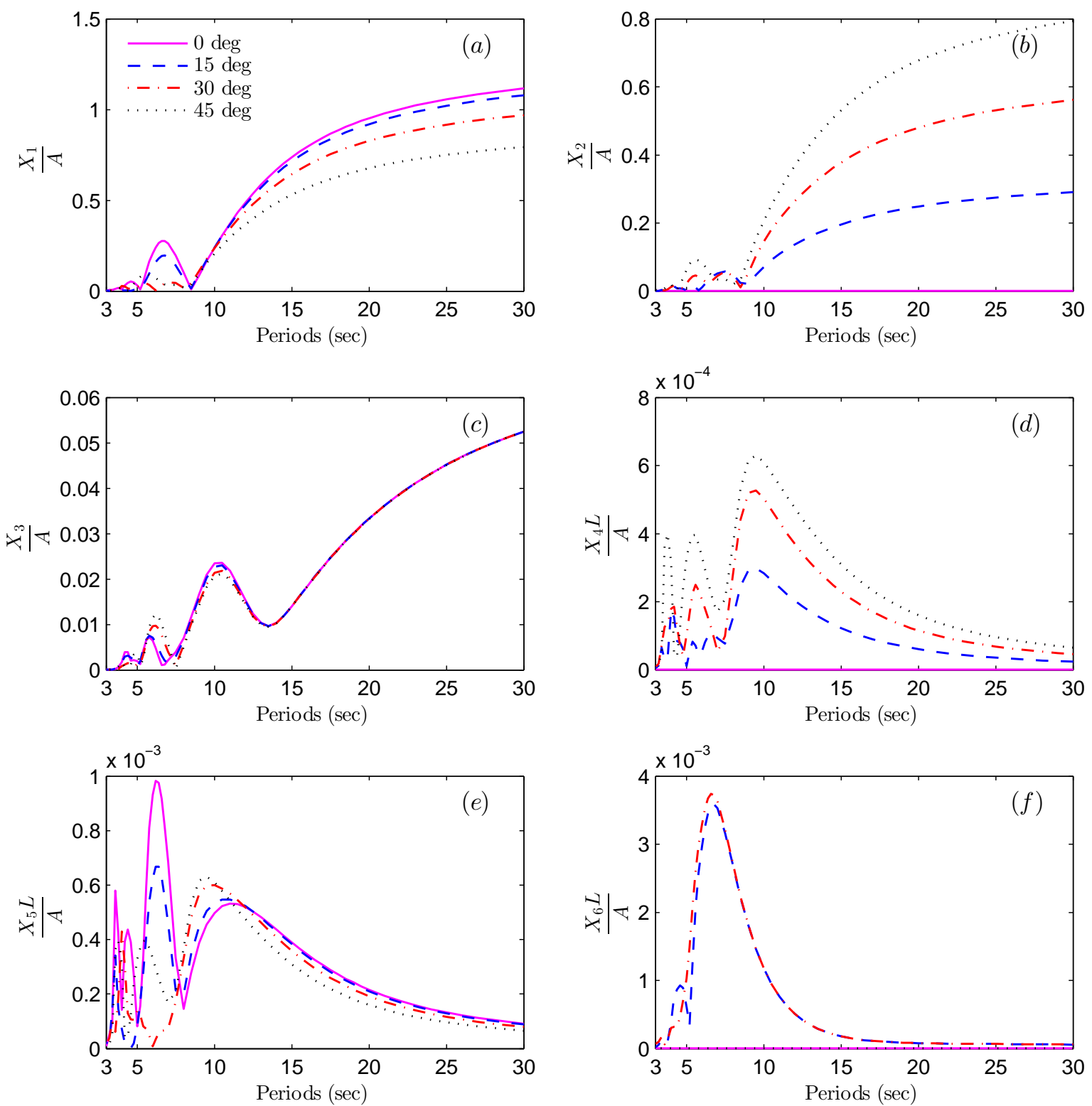

Figure 9: RAOs of 6 DoF in regular waves with different wave headings. (a) Surge; (b) Sway; $(c)$ Heave; $(d)$ Roll; $(e)$ Pitch; $(f)$ Yaw. $\mathrm{A}$ is the amplitude of incident wave; $\mathrm{L}$ is the characteristic length of the TLP. 


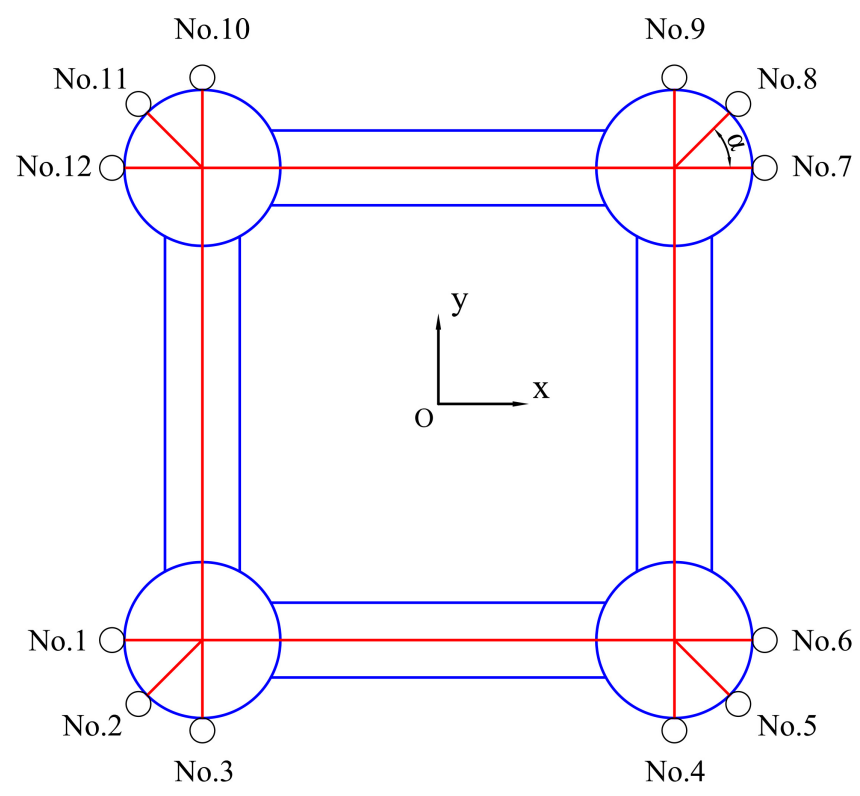

Figure 10: The arrangement plan of twelve tendons and the indexes. Each column is attached to three tendons and the angle between tendons is $\alpha=45^{\circ}$ in per corner.
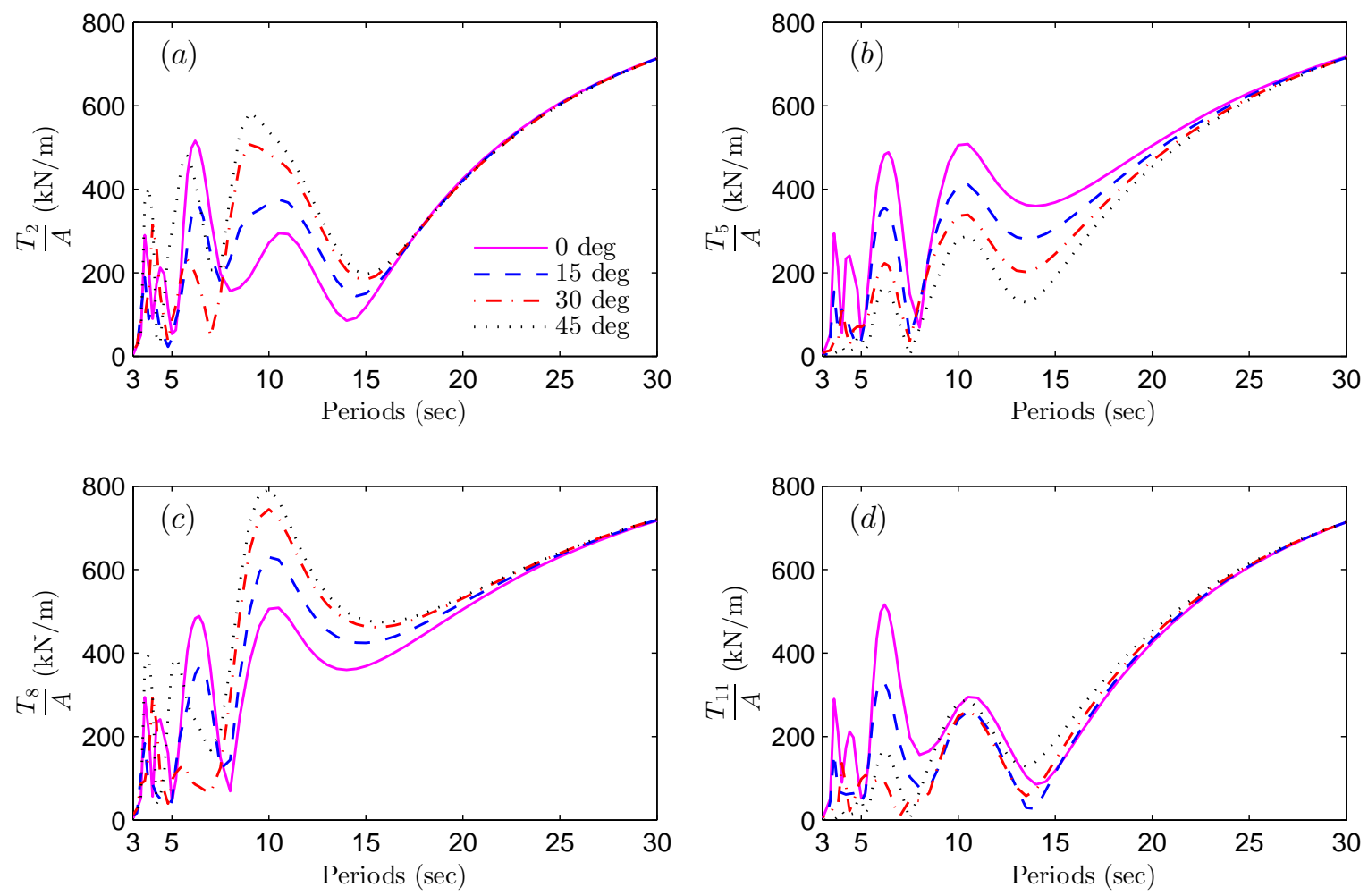

Figure 11: RAOs of different tendons in regular waves with different wave headings. (a) No.2 tendon; $(b)$ No.5 tendon; (c) No.8 tendon; (d) No.11 tendon. A is the amplitude of incident wave. 

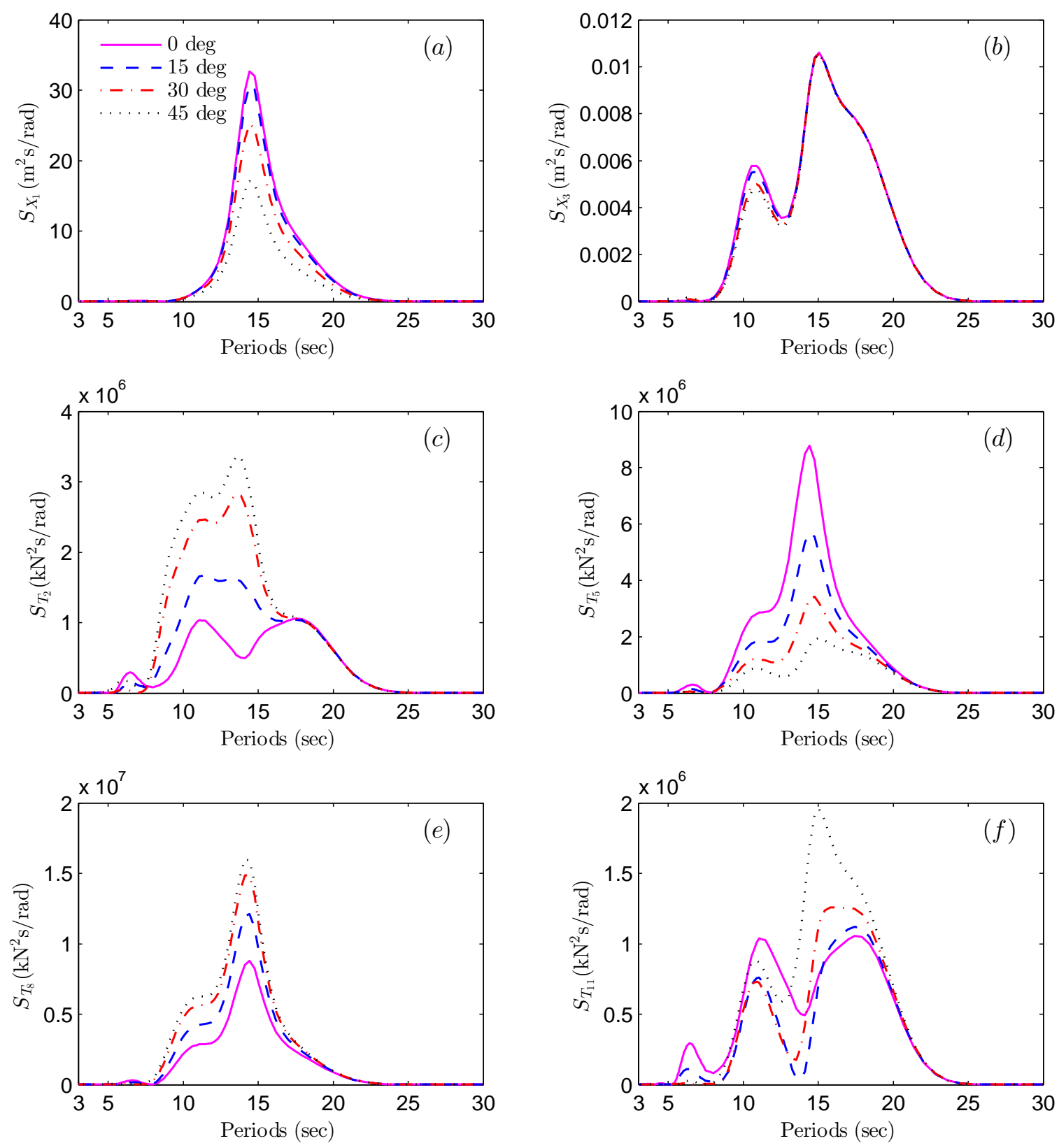

Figure 12: Response spectra in long-crest waves. (a) Surge; (b) Heave; $(c)$ No.2 tendon tension; $(d)$ No.5 tendon tension; $(e)$ No.8 tendon tension; $(f)$ No.11 tendon tension. The survival condition: $H_{s}=12.3 \mathrm{~m} ; T_{p}=14.4$ sec; $\gamma=3.3$; wave spreading function $=$ none. 
The response spectra in short-crest waves with a wave spreading function $\left(\cos ^{2}\right)$ are computed and presented in Figure 13.

The statistics of the motion and tension responses are listed in Table 5. Based on Rayleigh distribution, the MPM responses during 3-hour simulations are proportional to the root mean squares of the zero spectral moments. Table 5 shows that the maximum heave and surge motion responses occur at $0^{\circ}$ wave heading in long-crest wave. Because of the symmetry of the hull, the worst wave headings are $0^{\circ}, 90^{\circ}, 180^{\circ}$, and $270^{\circ}$. Compared with the dynamic tensions of the other three different tendons at each corner of the platform, the dynamic tension of No.8 tendon reaches the maximum in $45^{\circ}$ wave heading.

\begin{tabular}{c|c|c|c|c|c|c|c}
\multicolumn{7}{c}{ Table 5: The zero spectral moment $\left(m_{0}\right)$ of different responses in survival condition. } \\
\hline Wave & Heading & Heave & Surge & Tendon2 & Tendon5 & Tendon8 & Tendon11 \\
\hline Unit & $\mathrm{deg}$ & $\mathrm{m}^{2} \mathrm{~s} / \mathrm{rad}$ & $\mathrm{m}^{2} \mathrm{~s} / \mathrm{rad}$ & $\mathrm{kN}^{2} \mathrm{~s} / \mathrm{rad}$ & $\mathrm{kN}^{2} \mathrm{~s} / \mathrm{rad}$ & $\mathrm{kN}^{2} \mathrm{~s} / \mathrm{rad}$ & $\mathrm{kN}^{2} \mathrm{~s} / \mathrm{rad}$ \\
\hline Long crest & 0 & $2.32 \mathrm{E}-03$ & $3.48 \mathrm{E}+00$ & $3.82 \mathrm{E}+11$ & $1.44 \mathrm{E}+12$ & $1.44 \mathrm{E}+12$ & $3.82 \mathrm{E}+11$ \\
Long crest & 15 & $2.27 \mathrm{E}-03$ & $3.27 \mathrm{E}+00$ & $5.72 \mathrm{E}+11$ & $9.58 \mathrm{E}+11$ & $1.98 \mathrm{E}+12$ & $2.61 \mathrm{E}+11$ \\
Long crest & 30 & $2.18 \mathrm{E}-03$ & $2.68 \mathrm{E}+00$ & $8.59 \mathrm{E}+11$ & $6.20 \mathrm{E}+11$ & $2.52 \mathrm{E}+12$ & $2.71 \mathrm{E}+11$ \\
Long crest & 45 & $2.14 \mathrm{E}-03$ & $1.83 \mathrm{E}+00$ & $1.06 \mathrm{E}+12$ & $3.96 \mathrm{E}+11$ & $2.80 \mathrm{E}+12$ & $3.96 \mathrm{E}+11$ \\
\hline Short crest & 0 & $2.22 \mathrm{E}-03$ & $2.65 \mathrm{E}+00$ & $5.36 \mathrm{E}+11$ & $1.50 \mathrm{E}+12$ & $1.50 \mathrm{E}+12$ & $5.36 \mathrm{E}+11$ \\
Short crest & 15 & $2.22 \mathrm{E}-03$ & $2.53 \mathrm{E}+00$ & $5.99 \mathrm{E}+11$ & $1.17 \mathrm{E}+12$ & $1.79 \mathrm{E}+12$ & $5.02 \mathrm{E}+11$ \\
Short crest & 30 & $2.22 \mathrm{E}-03$ & $2.22 \mathrm{E}+00$ & $6.55 \mathrm{E}+11$ & $8.76 \mathrm{E}+11$ & $2.00 \mathrm{E}+12$ & $5.34 \mathrm{E}+11$ \\
Short crest & 45 & $2.22 \mathrm{E}-03$ & $1.79 \mathrm{E}+00$ & $6.77 \mathrm{E}+11$ & $6.57 \mathrm{E}+11$ & $2.07 \mathrm{E}+12$ & $6.57 \mathrm{E}+11$ \\
\hline
\end{tabular}

\subsection{Airgap computations}

The airgap in survival condition is a critical design criterion as it is correlated with wave slamming. The airgap is computed by Eqn.(10). With consideration of diffraction and radiation effects induced by floating body, wave elevations under the deck should be computed.

The convergence tests for wave elevations are carried out in order to ensure the validity of the present numerical method. The results of the convergence tests are illustrated in Figure 14(a). The predicted wave elevation at the probe $(21.5 \mathrm{~m}, 21.5 \mathrm{~m}, 0 \mathrm{~m})$ converges in the four different wave headings and becomes a constant as the number of panels increases over 3000. Thus, the mesh size is set to $2.5 \mathrm{~m}$, corresponding to approximately 3000 panels. The maximum wave elevations always occur around the columns, but the wave elevation does not converge as the distance between probe and column decreases. As illustrated in Figure 14(b), there are totally 9 probes placed near the column in order to determine the proper distance to the column for the following analyses. It can be seen that the maximum wave elevation occurs at $1 \mathrm{~m} \sim 1.25 \mathrm{~m}$ away from the column. When the distance to column is less than $1 \mathrm{~m}$, the wave elevation decreases instead, which is not realistic. Therefore, the nearest numerical wave probes for airgap prediction are placed $1 \mathrm{~m}$ away from the column.

As shown in Figure 15, totally 341 numerical wave probes are placed on the still water surface. Similar to other response analyses, the wave elevation RAOs with four different wave headings in regular waves are computed and plotted in Figure 16. The selected wave period is $11.5 \mathrm{sec}$ close to the zero-upcrossing period 

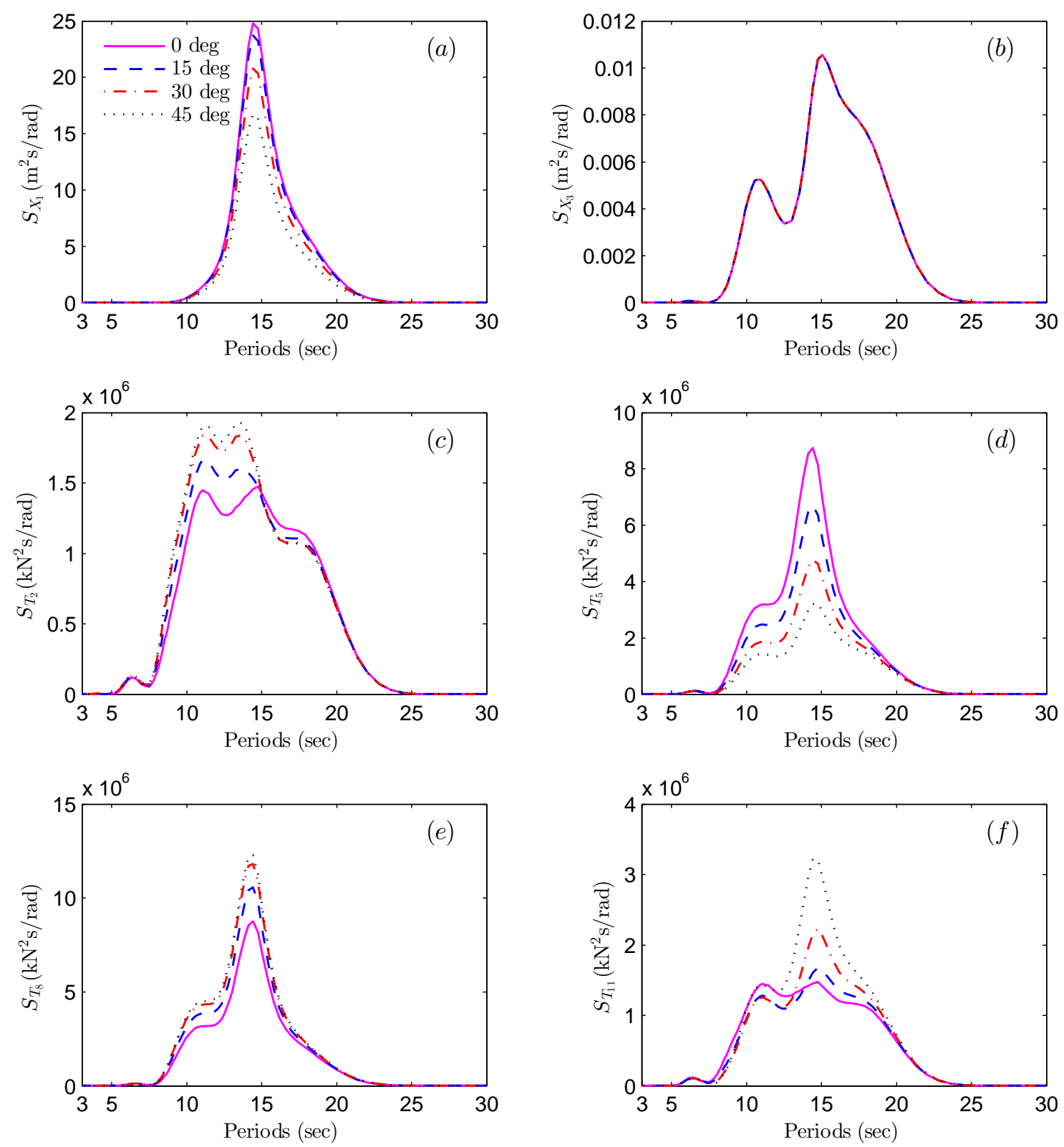

Figure 13: Response spectra in short-crest waves. (a) Surge; $(b)$ Heave; $(c)$ No.2 tendon tension; $(d)$ No.5 tendon tension; $(e)$ No.8 tendon tension; $(f)$ No.11 tendon tension. the survival condition: $H_{s}=12.3 \mathrm{~m} ; T_{p}=14.4$ sec; wave spreading function $=$ cos ${ }^{2}$. 

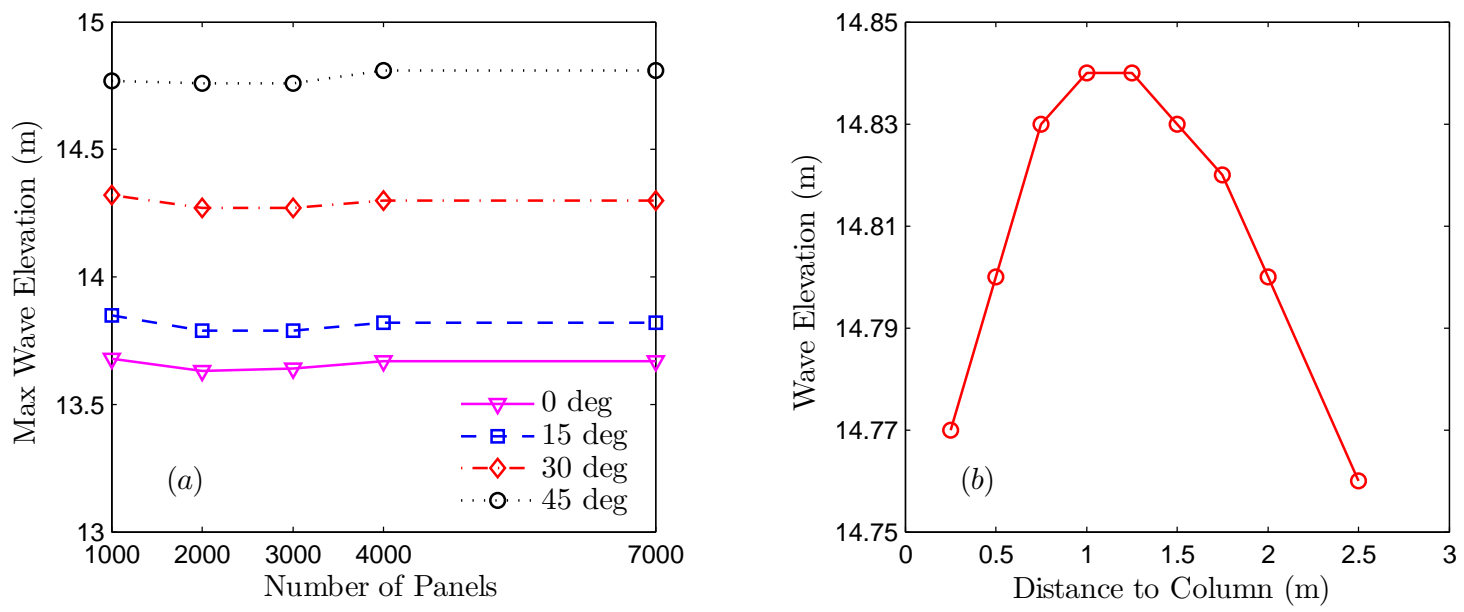

Figure 14: The convergence tests for estimating wave elevations. (a) Wave elevation at point $(21.5 \mathrm{~m}, 21.5 \mathrm{~m}, 0 \mathrm{~m})$ with increasing number of panels; $(b)$ Wave elevation with decreasing distance to the column.

of the survival condition. As shown in the contour plot, the maximum wave elevation, about 1.4 times of the incoming wave amplitude, occurs around the northeast column (NE Col). As shown in Figure 17, the 3-hour MPM responses of wave elevation in irregular waves are plotted in the contours. The maximum wave elevation is $14.7 \mathrm{~m}$.

It should be noted that the setdown of the TLP has a significant impact on airgap. When the platform has an offset, induced by mean horizontal environmental load, the draft of the platform will increase because of the restraint of tendons. The length of the tendons is assumed to be a constant when the offset is small relative to water depth. In general, the horizontal displacement of the platform is usually less than $5 \%$ of the water depth. In the present study, the maximum setdown is assumed to be $2 \mathrm{~m}$. As described in Eqn.(10), both the vertical motion of the platform and the wave elevation should be taken into account for airgap computations. The airgap contour is plotted in Figure 18. As presented, the minimum airgap occurs near the northeast column $(\mathrm{NE} \mathrm{Col})$. The reason is that the maximum wave elevation occurs there, and the vertical motion (heave, roll, and pitch) is very small so that it has a negligible impact on the airgap. As shown in Figure 18(d), the most probable minimum (MPM) airgap in survival condition is approximately $8.25 \mathrm{~m}$.

\section{Optimization Based on Surrogate Model}

\subsection{Objectives and constraints for optimization}

The maximum dynamic tendon tension and the total weight of the platform are selected as the two objectives. The maximum dynamic tendon tension represents the safety, and the total weight means the cost. The objective functions should be optimized for better safety and cost. In the previous section, the dynamic tension responses of four typical tendons in survival condition are analyzed and the MPM response occurs at No.8 tendon for $45^{\circ}$ wave heading. Therefore, only No.8 tendon is computed and analyzed during the optimization process. In the present study, three constraints are adopted including the maximum heave motion, the maximum surge motion, and the minimum airgap for functional requirements. Considering risers' limited compensation ability for motions, the heave motion should be less than $0.2 \mathrm{~m}$ and the surge motion should 


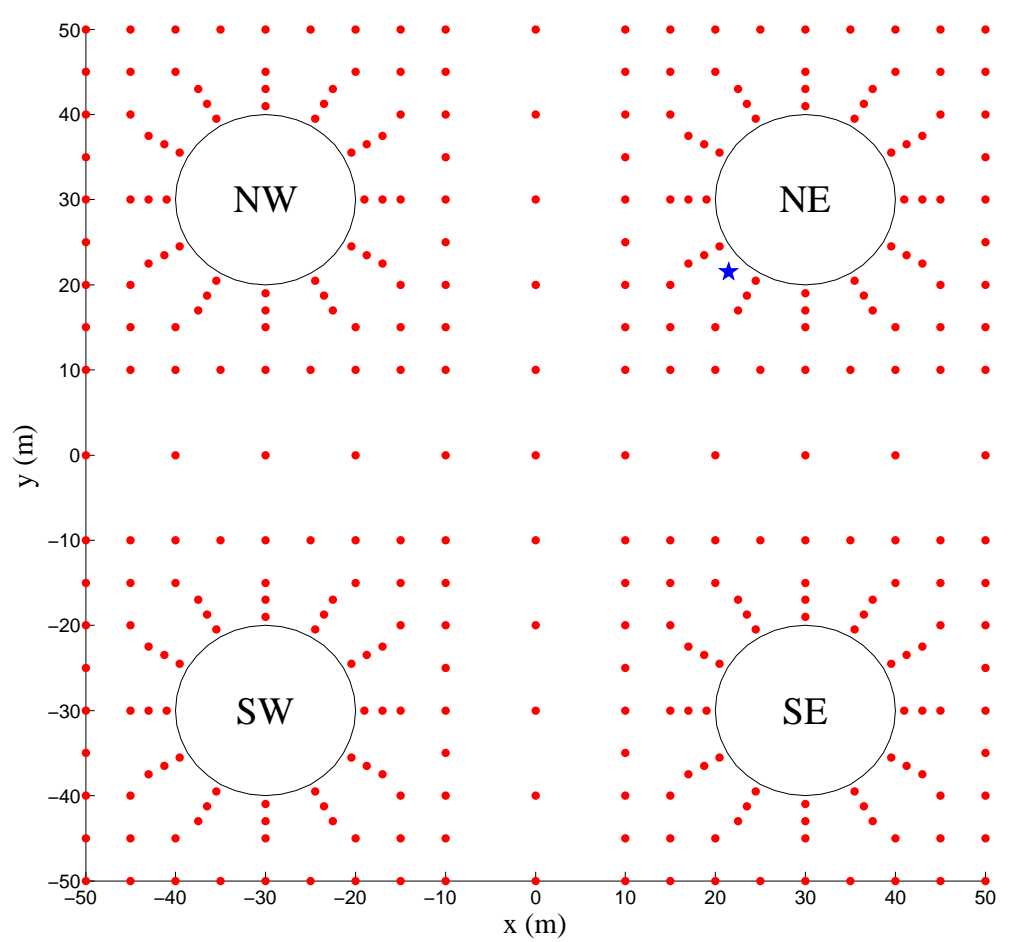

Figure 15: The arrangement plan of probes (red circles) for estimating the wave elevations on the still water surface $z=0$. The blue star symbol represents the point $(21.5 \mathrm{~m}, 21.5 \mathrm{~m}, 0 \mathrm{~m})$ used for airgap analysis in optimization section.

be less than $7 \mathrm{~m}$ in optimization module. To avoid wave slamming, the airgap should be larger than $8 \mathrm{~m}$ as the effects of the wind and current are neglected at the present stage. The minimum airgap induced by wave elevation and combined motion occurs around the northeast column (NE Col) for $45^{\circ}$ wave heading. Thus, in order to save computing time, the numerical wave probes are only arranged near the NE Col.

\subsection{Surrogate model}

The total weight of the platform is estimated based on the principal dimensions. However, the tendon tension responses, motion responses in 6 DoF, and airgap should be evaluated through direct numerical simulations. The RBF models are built to estimate the performances of different TLPs instead of numerical simulations. The major task for building a RBF model is to select a proper formal parameter to minimize the prediction error. The range of formal parameter is between 0 and 2 in general (Han, 2015). Through the leave-one-out cross validation (LOOCV), the prediction errors with different formal parameters are computed and listed in Table 6. The results are also illustrated in Figure 19. 
$(a)$

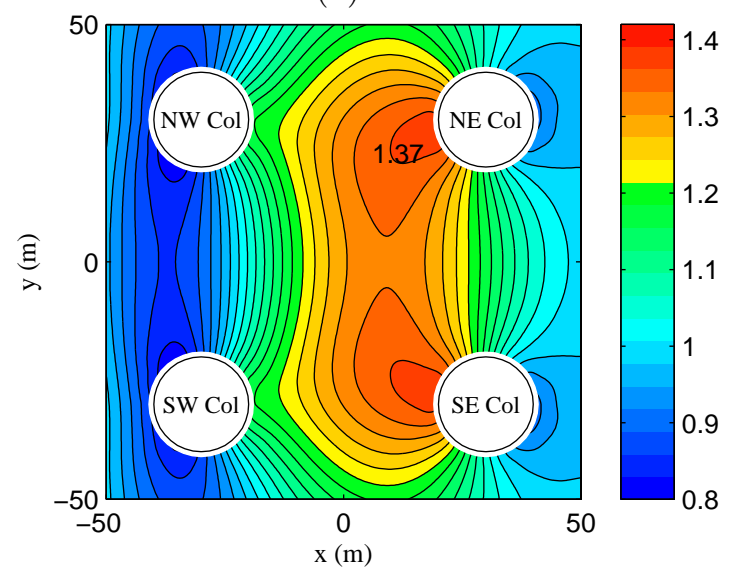

$(c)$

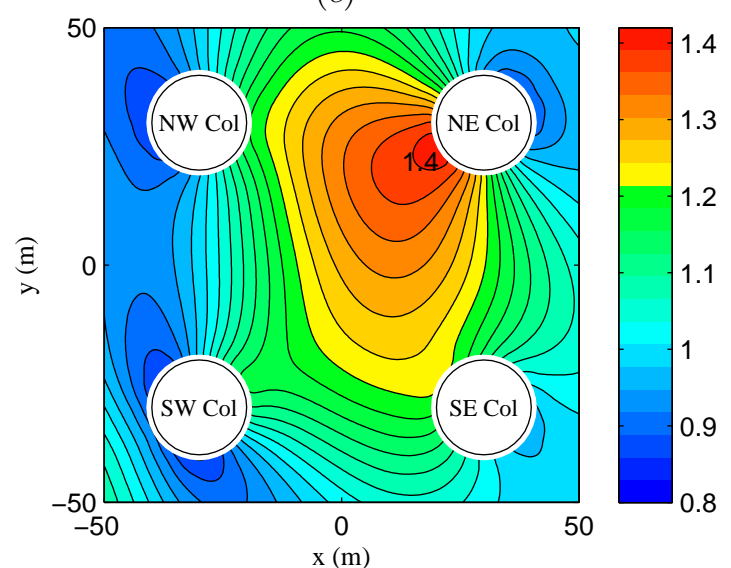

(b)

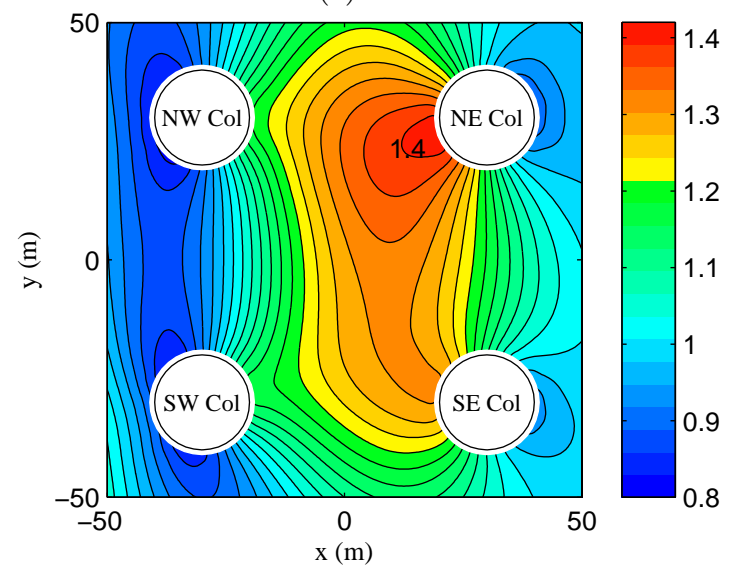

$(d)$

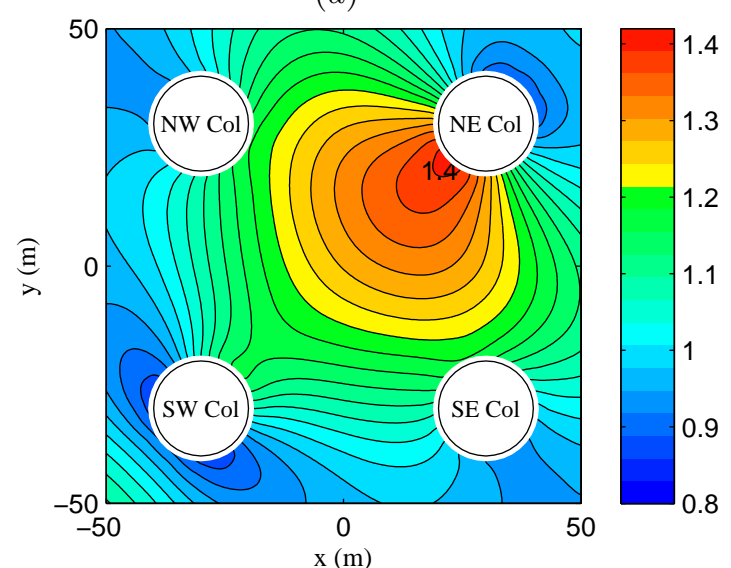

Figure 16: The contour of wave elevation RAOs in regular waves. The wave elevation is non-dimensionalized by incident wave amplitude. Wave heading: (a) $\beta=0^{\circ} ;(b) \beta=15^{\circ} ;(c) \beta=30^{\circ} ;(d) \beta=45^{\circ}$. Wave period $T=11.5$ sec. 
$(a)$

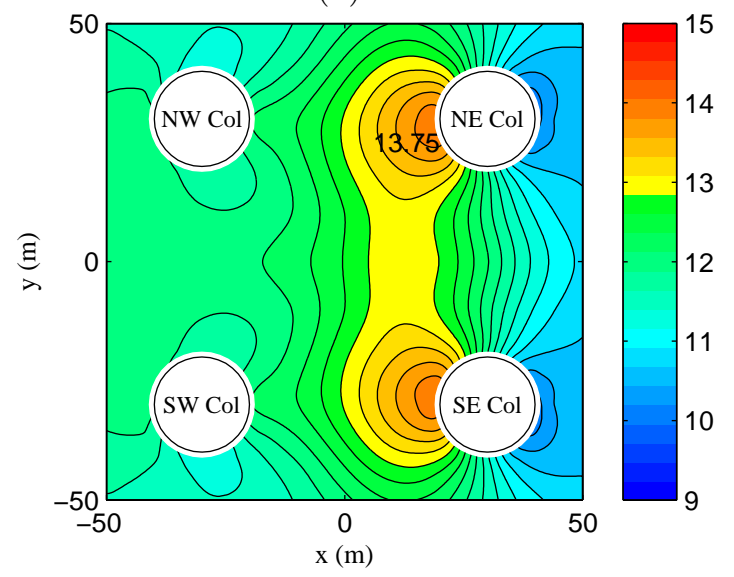

$(c)$

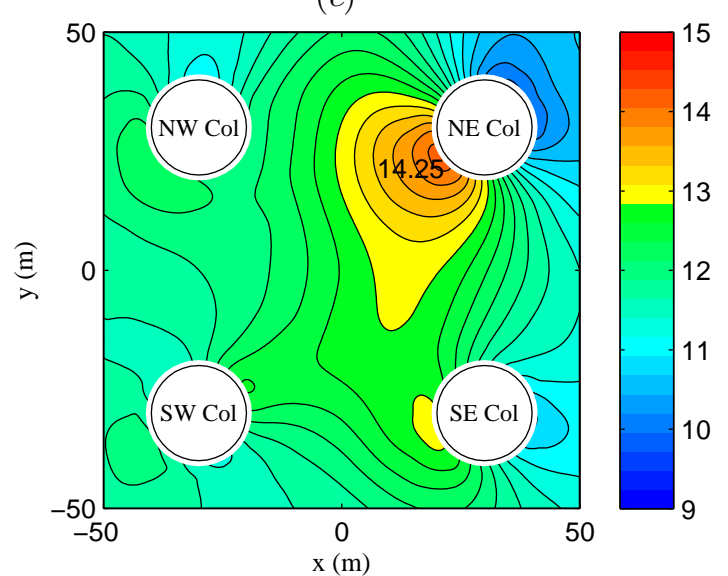

$(b)$

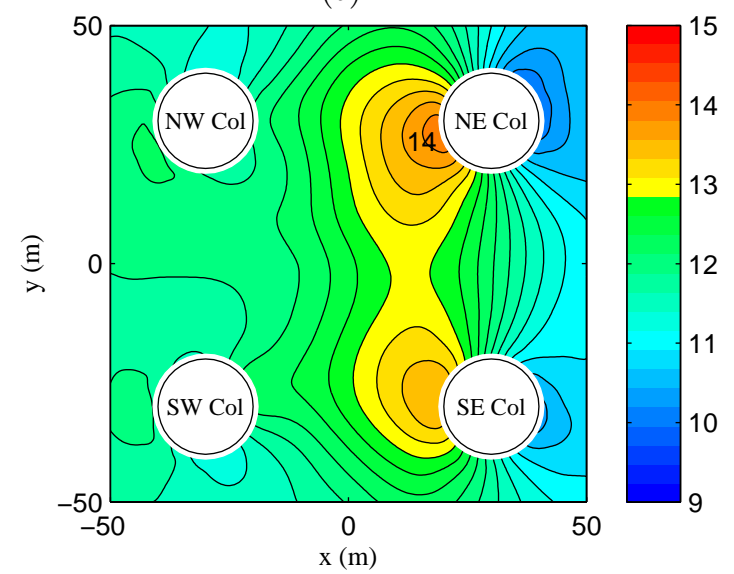

$(d)$

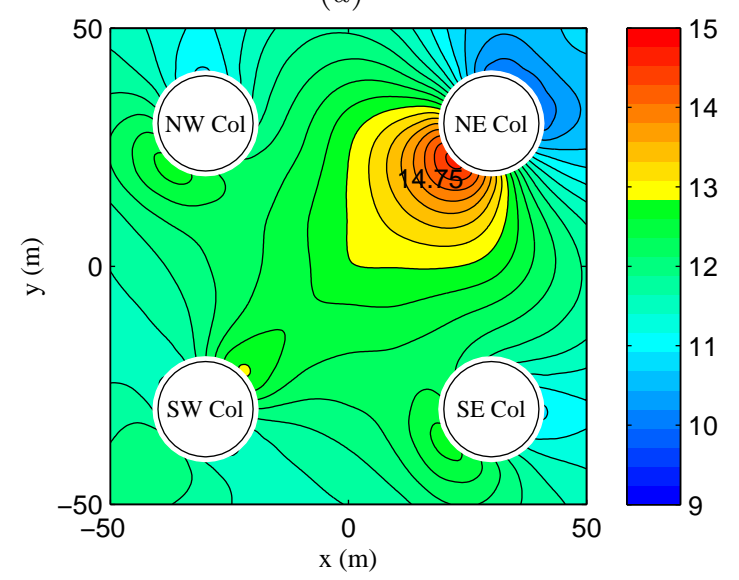

Figure 17: The contour of wave elevations (MPM in 3 hours) in long-crest waves. $H_{s}=12.3 \mathrm{~m} ; T_{p}=14.4$ sec; Spreading function $=$ none; Wave heading: (a) $\beta=0^{\circ}$; (b) $\beta=15^{\circ} ;(c) \beta=30^{\circ} ;(d) \beta=45^{\circ}$. 
(a)

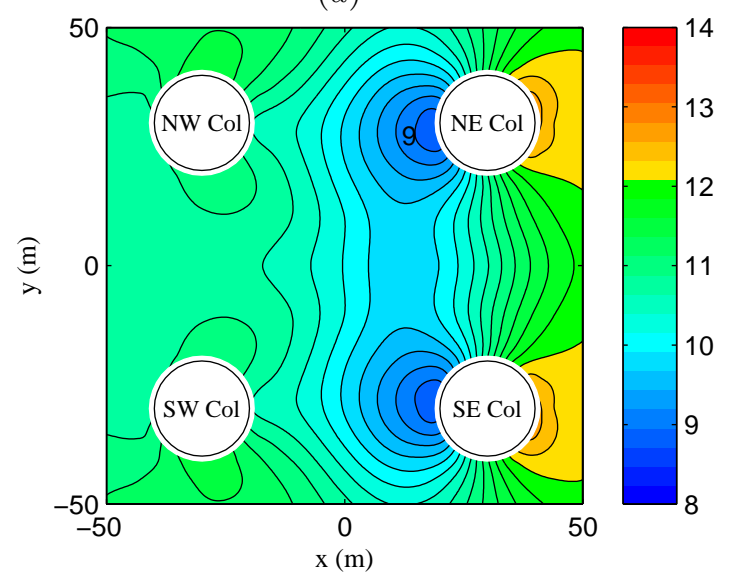

(c)

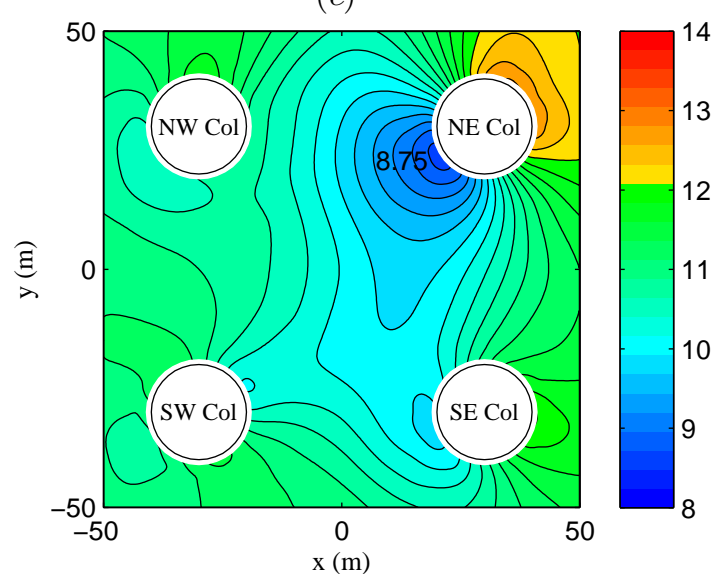

(b)

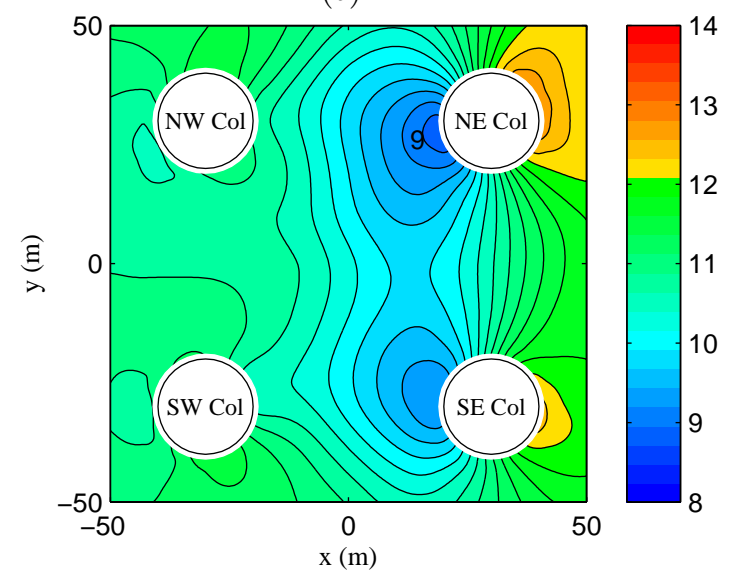

(d)

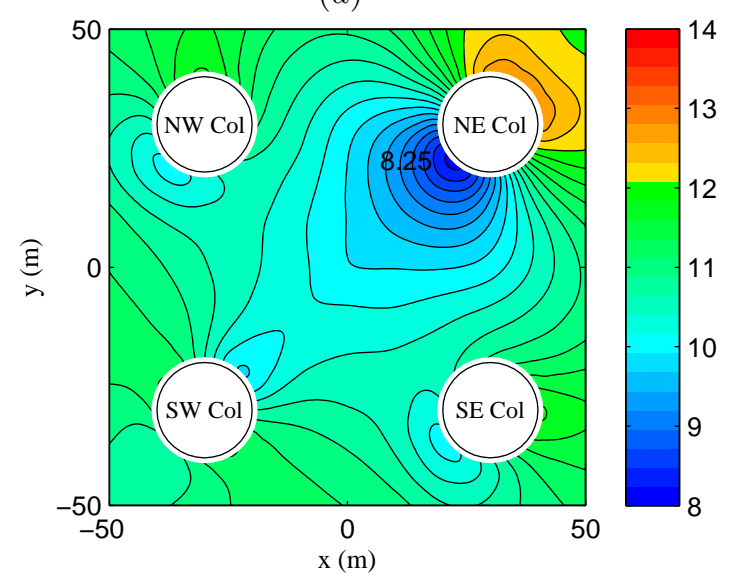

Figure 18: The contour of airgaps in long-crest waves. $H_{s}=12.3 \mathrm{~m} ; T_{p}=14.4 \mathrm{sec}$; Spreading function $=$ none; Wave heading: $(a)$ $\beta=0^{\circ} ;(b) \beta=15^{\circ} ;(c) \beta=30^{\circ} ;(d) \beta=45^{\circ}$. 

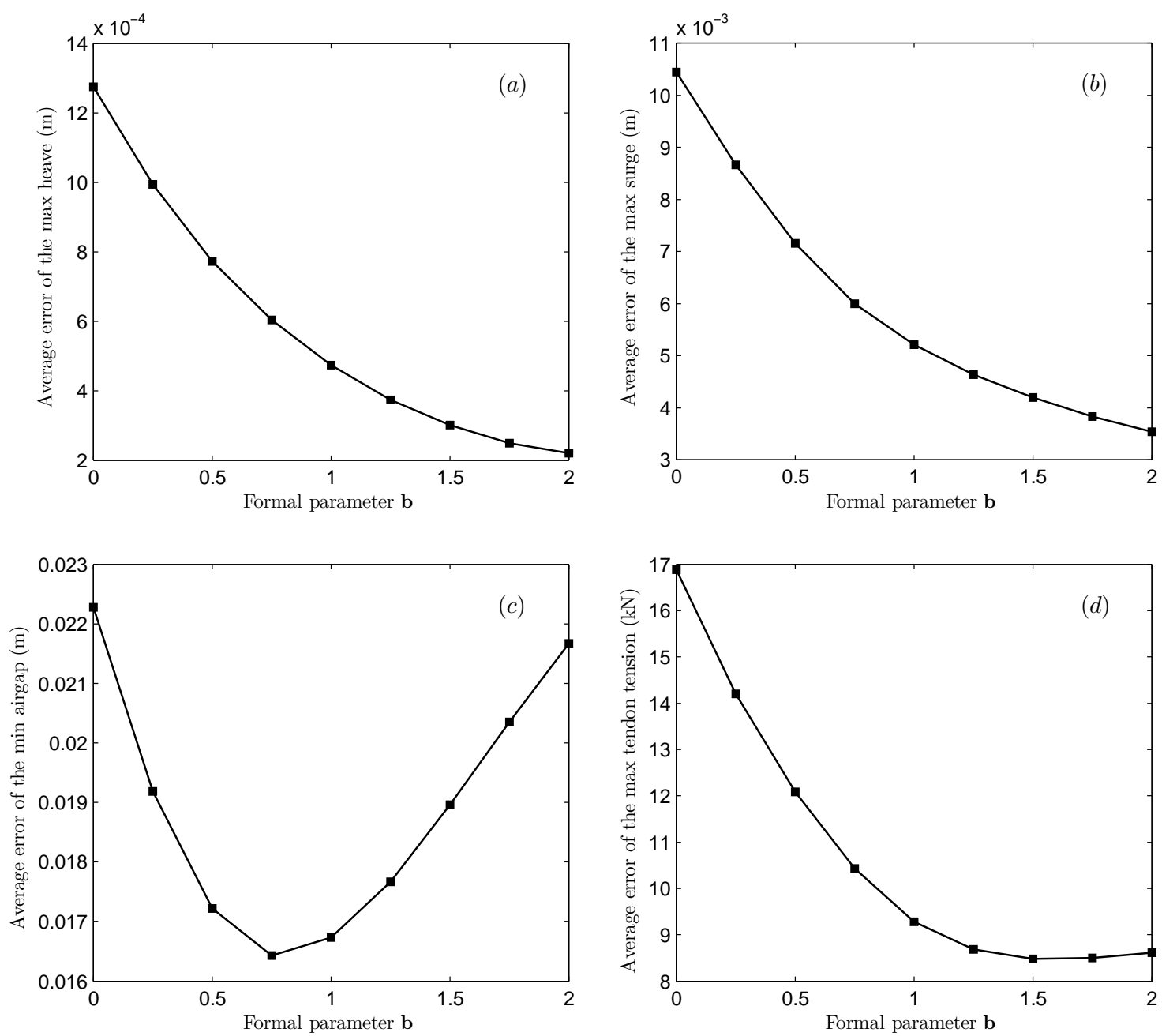

Figure 19: The average errors of RBF models by LOOCV with changing formal parameter. (a) Heave motion; (b) Surge motion; (c) Airgap; (d) Dynamic tendon tension. The maximum and minimum values are all MPM.

Table 6: The average errors of MPM with different formal parameters.

\begin{tabular}{ccccc}
\hline Formal parameter & Heave motion response & Surge motion response & Airgap & Tendon tension \\
\hline 0.00 & $1.27 \mathrm{E}-03 \mathrm{~m}$ & $1.04 \mathrm{E}-02 \mathrm{~m}$ & $2.23 \mathrm{E}-02 \mathrm{~m}$ & $16.88 \mathrm{kN}$ \\
0.25 & $9.94 \mathrm{E}-04 \mathrm{~m}$ & $8.66 \mathrm{E}-03 \mathrm{~m}$ & $1.92 \mathrm{E}-02 \mathrm{~m}$ & $14.20 \mathrm{kN}$ \\
0.50 & $7.72 \mathrm{E}-04 \mathrm{~m}$ & $7.16 \mathrm{E}-03 \mathrm{~m}$ & $1.72 \mathrm{E}-02 \mathrm{~m}$ & $12.08 \mathrm{kN}$ \\
0.75 & $6.04 \mathrm{E}-04 \mathrm{~m}$ & $6.00 \mathrm{E}-03 \mathrm{~m}$ & $1.64 \mathrm{E}-02 \mathrm{~m}$ & $10.43 \mathrm{kN}$ \\
1.00 & $4.73 \mathrm{E}-04 \mathrm{~m}$ & $5.21 \mathrm{E}-03 \mathrm{~m}$ & $1.67 \mathrm{E}-02 \mathrm{~m}$ & $9.28 \mathrm{kN}$ \\
1.25 & $3.73 \mathrm{E}-04 \mathrm{~m}$ & $4.64 \mathrm{E}-03 \mathrm{~m}$ & $1.77 \mathrm{E}-02 \mathrm{~m}$ & $8.68 \mathrm{kN}$ \\
1.50 & $3.00 \mathrm{E}-04 \mathrm{~m}$ & $4.20 \mathrm{E}-03 \mathrm{~m}$ & $1.90 \mathrm{E}-02 \mathrm{~m}$ & $8.48 \mathrm{kN}$ \\
1.75 & $2.49 \mathrm{E}-04 \mathrm{~m}$ & $3.84 \mathrm{E}-03 \mathrm{~m}$ & $2.04 \mathrm{E}-02 \mathrm{~m}$ & $8.50 \mathrm{kN}$ \\
2.00 & $2.19 \mathrm{E}-04 \mathrm{~m}$ & $3.54 \mathrm{E}-03 \mathrm{~m}$ & $2.17 \mathrm{E}-02 \mathrm{~m}$ & $8.62 \mathrm{kN}$ \\
\hline
\end{tabular}

As shown in Figure 19, the best formal parameters of RBF models for evaluating heave, surge, airgap, and tendon tension are 2.0, 2.0, 0.75, and 1.5, respectively. After determining the formal parameters of different 

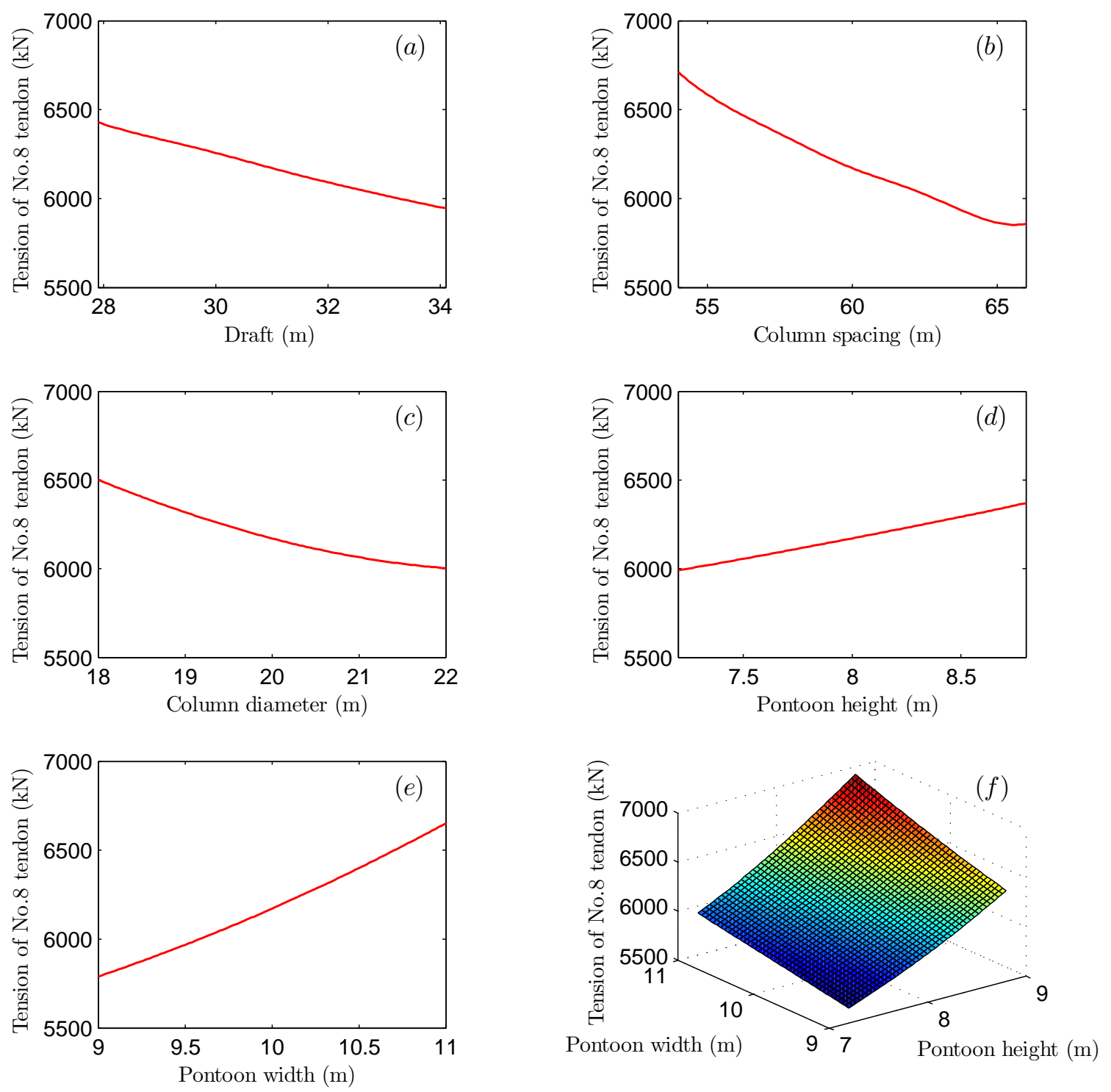

Figure 20: The variations of the maximum dynamic tendon tension with total 5 design variables based on RBF model. (a) Draft; (b) Column spacing; (c) Column diameter; $(d)$ Pontoon height; $(e)$ Pontoon width; $(f)$ Pontoon width and height.

RBF models, the performances of the TLPs with different hull forms can be computed using surrogate models. As illustrated in Figure 20, the variation tendencies of the tendon tension response are obtained by varying one dimension while the other four keeping the same. As can be observed, the maximum dynamic tendon tension decreases when the draft, column spacing, or column diameter increase. In contrast, the maximum dynamic tension has a positive correlation with pontoon height and width.

\subsection{Pareto-optimal solutions}

Based on the established surrogate models, NSGAII is employed for optimization, in which the population size and maximum generation are both set to 100. Finally, totally 30 individuals, which are non-dominated of each other, are found to form Pareto-optimal solutions. The optimal designs are sorted and ranked with the increase of tendon tension and the decrease of total weight, as listed in Table 7. 
Table 7: The Pareto-optimal solutions obtained by utilizing NSGAII.

\begin{tabular}{|c|c|c|c|c|c|c|c|}
\hline & Draft & $C S$ & $C D$ & $P H$ & $P W$ & $T_{\max }$ & $W_{T}$ \\
\hline Unit & $\mathrm{m}$ & $\mathrm{m}$ & $\mathrm{m}$ & $\mathrm{m}$ & $\mathrm{m}$ & $10^{3} \mathrm{kN}$ & $10^{4}$ ton \\
\hline 01 & 34.08 & 65.65 & 21.75 & 7.20 & 9.03 & 4.92 & 4.08 \\
\hline 02 & 34.01 & 65.46 & 21.41 & 7.25 & 9.03 & 4.93 & 4.03 \\
\hline 03 & 34.02 & 65.60 & 21.16 & 7.25 & 9.04 & 4.94 & 3.99 \\
\hline 04 & 33.97 & 65.55 & 20.71 & 7.24 & 9.03 & 4.97 & 3.92 \\
\hline 05 & 33.94 & 65.45 & 20.43 & 7.22 & 9.06 & 4.99 & 3.88 \\
\hline 06 & 34.08 & 65.51 & 20.02 & 7.22 & 9.03 & 5.00 & 3.82 \\
\hline 07 & 33.94 & 65.40 & 19.77 & 7.27 & 9.03 & 5.04 & 3.79 \\
\hline 08 & 33.82 & 65.54 & 19.48 & 7.25 & 9.04 & 5.06 & 3.74 \\
\hline 09 & 33.82 & 65.53 & 19.28 & 7.23 & 9.03 & 5.07 & 3.72 \\
\hline 10 & 33.90 & 65.49 & 18.90 & 7.25 & 9.03 & 5.10 & 3.67 \\
\hline 11 & 33.74 & 65.06 & 18.76 & 7.25 & 9.03 & 5.14 & 3.64 \\
\hline 12 & 33.79 & 65.28 & 18.29 & 7.22 & 9.03 & 5.18 & 3.58 \\
\hline 13 & 33.45 & 65.11 & 18.21 & 7.23 & 9.02 & 5.23 & 3.56 \\
\hline 14 & 33.30 & 64.65 & 18.14 & 7.23 & 9.01 & 5.28 & 3.55 \\
\hline 15 & 32.08 & 64.97 & 18.21 & 7.23 & 9.04 & 5.36 & 3.54 \\
\hline 16 & 31.12 & 64.72 & 18.15 & 7.22 & 9.03 & 5.43 & 3.51 \\
\hline 17 & 29.03 & 65.28 & 18.16 & 7.22 & 9.02 & 5.53 & 3.47 \\
\hline 18 & 28.24 & 64.03 & 18.07 & 7.21 & 9.01 & 5.68 & 3.44 \\
\hline 19 & 27.99 & 63.57 & 18.06 & 7.20 & 9.00 & 5.75 & 3.43 \\
\hline 20 & 28.11 & 62.69 & 18.07 & 7.24 & 9.01 & 5.82 & 3.43 \\
\hline 21 & 28.02 & 60.44 & 18.01 & 7.21 & 9.00 & 5.96 & 3.40 \\
\hline 22 & 28.07 & 59.57 & 18.01 & 7.21 & 9.00 & 6.01 & 3.40 \\
\hline 23 & 28.00 & 58.89 & 18.00 & 7.20 & 9.00 & 6.08 & 3.39 \\
\hline 24 & 28.17 & 57.03 & 18.05 & 7.21 & 9.00 & 6.20 & 3.39 \\
\hline 25 & 28.08 & 56.10 & 18.00 & 7.20 & 9.00 & 6.28 & 3.37 \\
\hline 26 & 27.98 & 55.42 & 18.00 & 7.20 & 9.00 & 6.34 & 3.36 \\
\hline 27 & 28.16 & 54.62 & 18.00 & 7.21 & 9.00 & 6.41 & 3.36 \\
\hline 28 & 27.90 & 54.00 & 18.00 & 7.20 & 9.00 & 6.53 & 3.35 \\
\hline 29 & 27.90 & 54.00 & 18.00 & 7.20 & 9.00 & 6.53 & 3.35 \\
\hline 30 & 27.90 & 54.00 & 18.00 & 7.20 & 9.00 & 6.53 & 3.35 \\
\hline
\end{tabular}

Meanwhile, the optimization results are verified by direct numerical simulations to ensure the accuracy of the surrogate model. The comparison of optimization results with the simulated ones and all sample points are illustrated in Figure 21. The rhombus symbol in the plot represents the initial design. The vertical and horizontal axes passing that point divide the plot into four regions. The points shown in bottom left region mean 


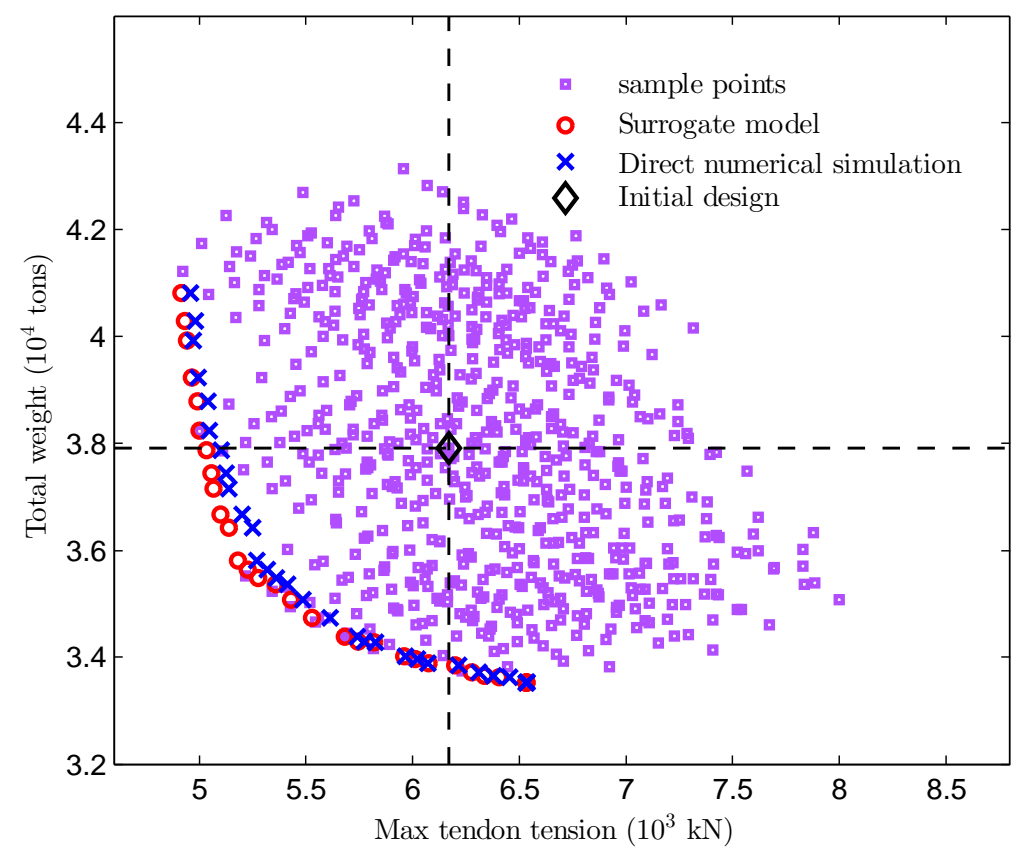

Figure 21: 30 Pareto-optimal solutions obtained by NSGAII based on RBF model and compared with direct numerical simulations.

that the designs are better than the initial design in terms of the two objectives, total weight and maximum tendon tension. The top left and bottom right regions are much better than the initial design in terms of dynamic tendon tension and total weight, respectively.

By comparing the results by surrogate model and numerical simulations, it shows that the maximum relative errors are less than $2.5 \%$, as illustrated in Figure 22. This confirms the surrogate model can provide results with satisfactory accuracy. Moreover, the constraints of the Pareto-optimal solutions are all computed and illustrated in Figure 23. As shown, all constraints are fulfilled for the functional requirements.

Based on the 30 Pareto-optimal solutions obtained by optimization procedure, the variation tendencies of five design variables are illustrated in Figure 24. The $x$-axis represents the number of the Pareto-optimal solutions. As the number grows, the dynamic tendon tension increases while the total weight decreases. As shown in Figure 24(d) and (e), both the pontoon width and height maintain at the lower bound among 30 Paretooptimal solutions. This suggests decreasing pontoon width and height can not only decrease the dynamic tendon tension but also reduce the total weight, which is consistent with the results by surrogate model shown in Figure $20(d)$ and $(e)$. Different from pontoon width and height, increasing draft, column spacing, and column diameter can help reduce the dynamic tendon tension. This can also be observed in Figure 20(a), (b), and (c). The three design variables, including draft, column spacing, and column diameter, are all non-dimensionalized by the principle dimensions of initial design, and their variation tendencies are illustrated in Figure $24(f)$. As shown, the three design variables change in sequence. As the dynamic tendon tension decreases, the column spacing first increases from the lower bound to the upper bound. Then, the draft starts to increase. When both column spacing and draft reach their upper bound, the column diameter begins to increase from the lower bound to the upper bound. In order to validate the optimization results, dynamic tendon tension RAOs of the initial design and case No.1, No.11, No.21, and No.30 Pareto-optimal solutions are compared in Figure 25. The 


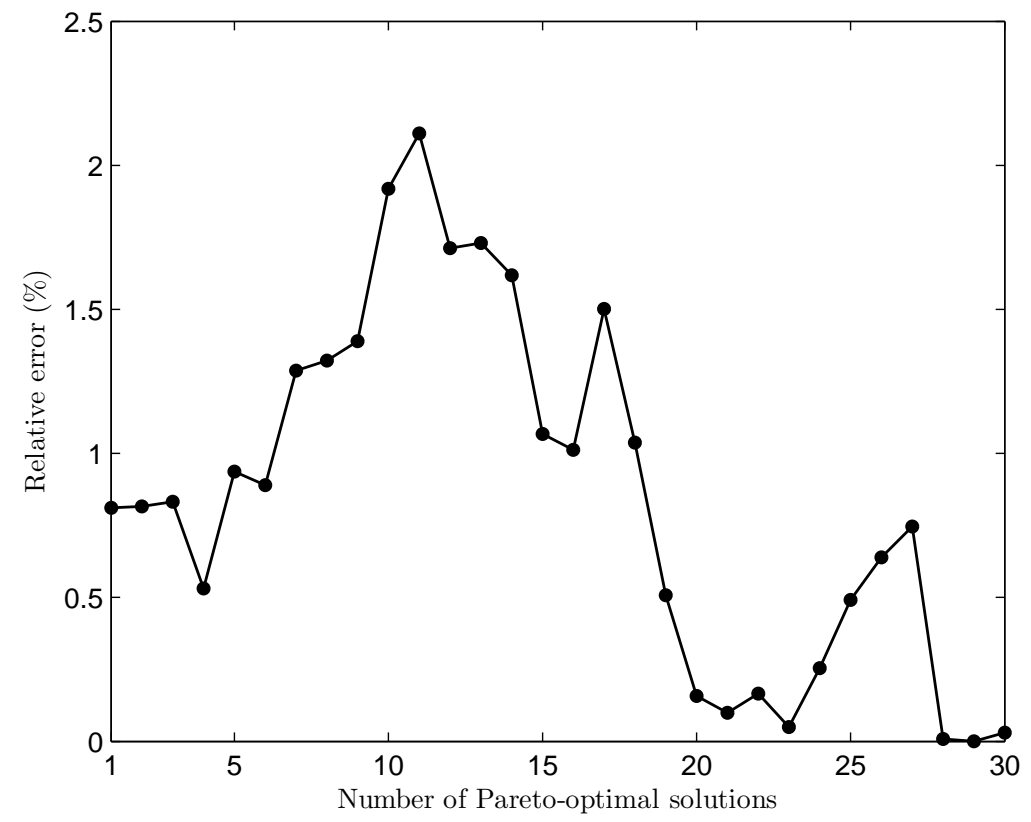

Figure 22: The relative error between the predictions using surrogate model and direct numerical simulations.

$(a)$

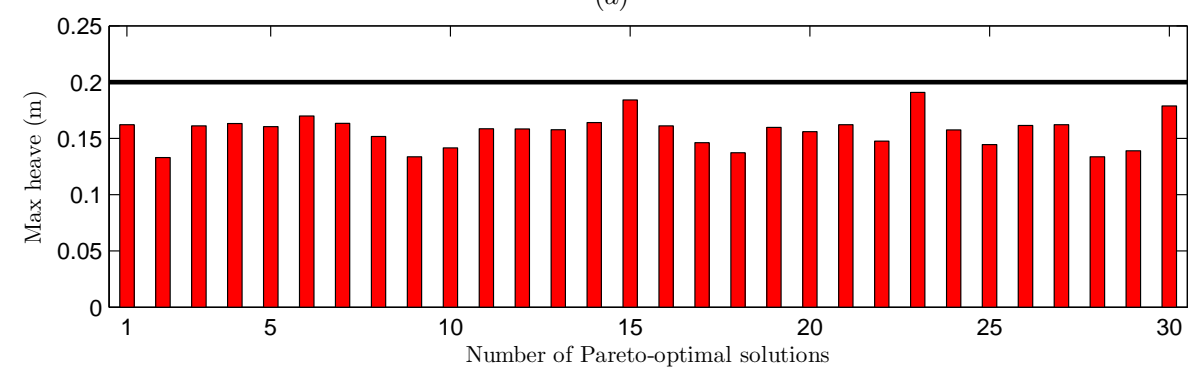

(b)

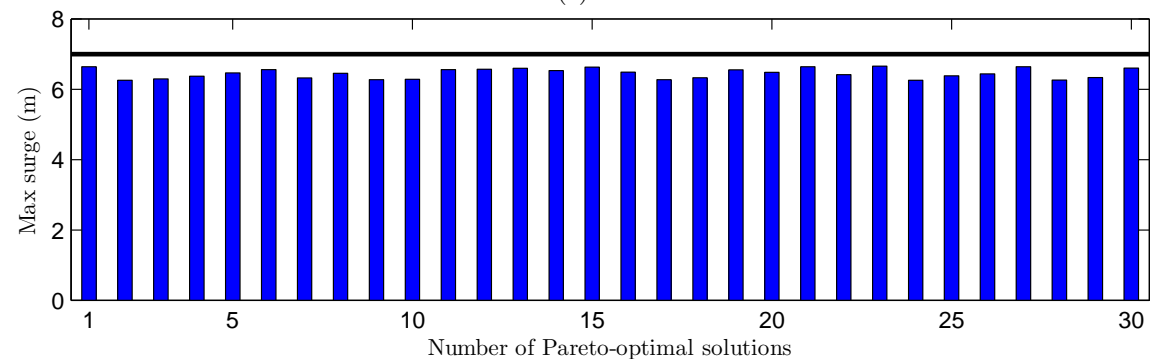

$(c)$

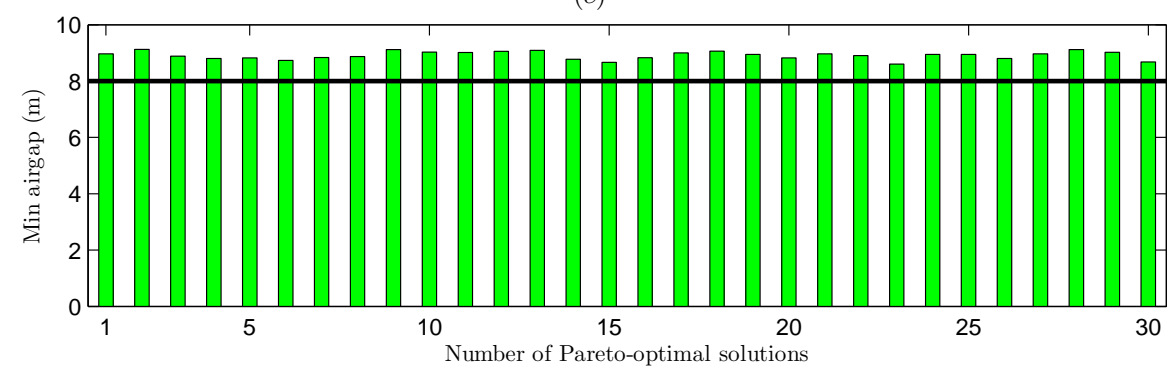

Figure 23: The constraint values of 30 Pareto-optimal solutions. (a) Maximum heave motion must less than 0.2 m; (b) Maximum surge motion must less than $7 \mathrm{~m}$; (c) Minimum airgap must more than $8 \mathrm{~m}$. 

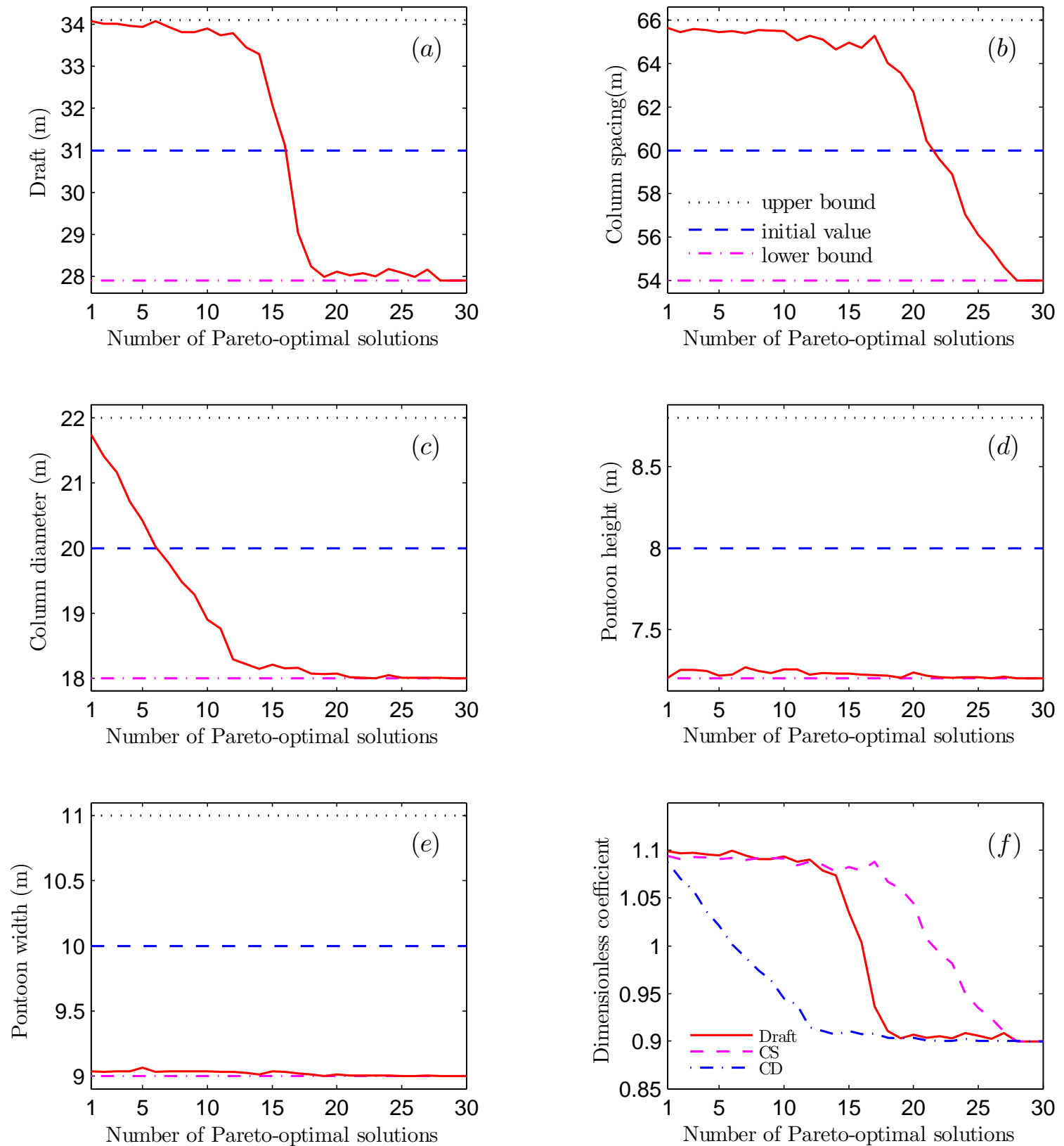

Figure 24: The variation of five design variables with 30 Pareto-optimal solutions. (a) Draft; (b) Column spacing; (c) Column diameter; $(d)$ Pontoon height; $(e)$ Pontoon width; $(f)$ Non-dimensional draft, column spacing, and column diameter. 

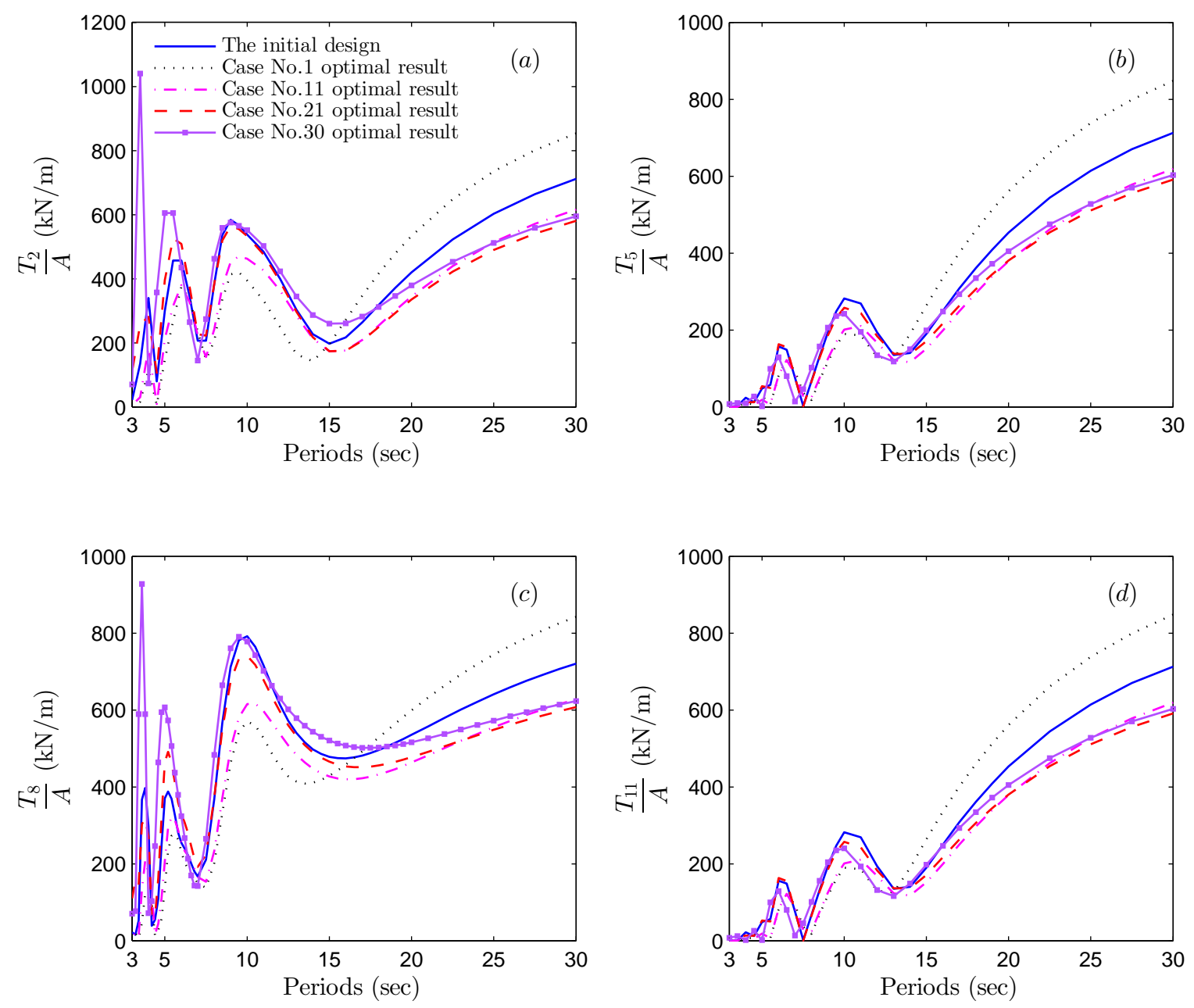

Figure 25: Tendon tension RAOs of four Pareto-optimal solutions and the initial design. (a) No.2 tendon; (b) No.5 tendon; (c) No.8 tendon; (d) No.11 tendon. The maximum tendon tension of Case No.1 optimal design: 4920 kN; Case No.11 optimal design: 5140 kN; Case No.21 optimal design: 5960 kN; Case No.30 optimal design: 6530 kN; initial design: 6170 kN.

third peak affects the performance of the TLP greatly because most of wave energy concentrates between wave period $10 \mathrm{sec}$ and $15 \mathrm{sec}$. In this region, the maximum dynamic tension of No.8 tendon is the largest compared with the others. As shown in Figure 25, the maximum tendon tension becomes larger as case number increases. Although case No.30 seems to converge to the initial design value, its weight is much smaller than the initial one as shown in Table 3 and Table 7. Therefore, the proposed method is superior.

Based on the above analyses, the most efficient modification strategy for decreasing the dynamic tendon tension is found. Larger column spacing should be considered first in order to decrease the maximum dynamic tendon tension. When the column spacing can not be increased due to functional limits, the draft and column diameter should be increased in sequence. Following the above mentioned design modification strategy, the total weight of the TLP can be minimized most efficiently than the other sequences of design modification. Moreover, it is suggested that the pontoon width and height should be sized as small as possible with considering the other design criteria, such as the minimum pontoon volume for ballast water requirement. 


\section{Conclusions}

An Innovative TLP Optimization Program (ITOP) has been developed to solve the multi-objective optimization problem for a TLP. Hull draft, column spacing, column diameter, pontoon height, and pontoon width are selected as design variables. The objective functions include the maximum dynamic tendon tension and the total weight of the platform, with three constraints being the maximum heave motion, the maximum surge motion, and the minimum airgap. A surrogate model based on radial basis function (RBF) has been built and adopted to estimate the hydrodynamic performances of the TLPs with different hull forms. A multi-objective evolutionary algorithm, Non-dominated Sorting Genetic Algorithm II (NSGAII), is applied to select for the Pareto-optimal solutions.

The hydrodynamic behavior of the TLP is studied first. By examining the numerical results, it is revealed that:

I) The maximum heave and surge motion responses occur in $0^{\circ}$ wave heading in long-crest waves.

II) The MPM dynamic tendon tension occurs at No.8 tendon in $45^{\circ}$ wave heading in survival condition.

III) The minimum airgap occurs near the NE Col because the maximum wave elevation occurs there and the vertical motion of the platform is very small.

Multi-objective optimization based on surrogate model is performed for the TLP. It is found that:

I) The maximum dynamic tension shows positive correlation with pontoon height and width, but negative correlation with draft, column spacing, and column diameter.

II) The most efficient design modification strategy is found to suppress the dynamic tendon tension. As found, the column spacing, draft, and column diameter should be increased in sequence. By this strategy, the dynamic tendon tensions can be reduced and the total structural weight of the platform can be minimized as much as possible.

\section{Acknowledgement}

The authors would like to express sincere thanks to Professor Chi Yang for her valuable suggestions and discussions. Thanks also go to the anonymous reviewers for their valuable comments and suggestions, which greatly enhanced the quality of this article. The first author (X. Z.) gratefully acknowledges the financial support from Chinese government through the 1000-Young Talent Program with Grant No. 15Z127060020, and National Natural Science Foundation of China with Grant No. 51679138. The financial support from National Basic Research of China with Grant No. 2015CB251203 and No. 2013 CB036103 is also gratefully acknowledged. Partial financial support is provided by the Chun Tsung Program through Shanghai Jiao Tong University.

\section{Reference}

Akagi, S., Yokoyama, R., Ito, K., 1984. Optimal design of semisubmersible's form based on systems analysis. J. Mechanisms, Transmissions, and Automation in Design 106 (4), 524-530.

Anderson, M. J., Whitcomb, P. J., 1970. Design of experiments. Concise Encyclopedia of Statistics 2 (Sept), $33-53$. 
API RP 2MET, 2014. Derivation of Metocean Design and Operating Conditions. In: First Edition.

API RP 2SK, 2005. Recommended Practice for Design and Analysis of Stationkeeping Systems for Floating Structures. In: Third Edition.

API RP 2T, 1997. Recommended Practice for Planning, Design and Constructoing Tension Leg Platforms. In: Second Edition.

Audet, C., Booker, A. J., Dennis, J. E., Frank, P. D., Moore, D., 2000. A surrogate-model-based method for constrained optimization. American Institute of Aeronautics and Astronautics.

Birk, L., 2009. Application of constrained multi-objective optimization to the design of offshore structure hulls. Journal of Offshore Mechanics and Arctic Engineering 131 (1), 011301.

Birk, L., Clauss, G. F., Lee, J. Y., 2004. Practical application of global optimization to the design of offshore structures. In: Proceedings of the 23rd the International Conference on Offshore Mechanics and Arctic Engiineering, OMAE2004-51225, Vancouver, British Columbia.

Buhmann, M. D., 2009. Radial basis functions. Acta numerica 9, 1-38.

Clauss, G. F., Birk, L., 1996. Hydrodynamic shape optimization of large offshore structures. Applied Ocean Research 18 (4), 157-171.

Deb, K., 2001. Multi-objective Optimisation Using Evolutionary Algorithms: An Introduction. John Wiley and Sons, Inc.

Deb, K., 2011. Multi-Objective Optimization Using Evolutionary Algorithms. Wiley Press.

DNV, 2007. POSTRESP:Postprocessor for Statistical Response Calculations. SESAM User Manual.

DNV, 2013. WADAM: Wave Analysis by Diffraction and Morison Theory. SESAM User Manual.

Forrester, A. I. J., Keane, A. J., 2009. Recent advances in surrogate-based optimization. Progress in Aerospace Sciences $45(1), 50-79$.

Han, X., 2015. Numerical Simulation-based Design: Theory and Methods. Science Press.

Kim, J. D., Jang, B. S., 2016. Application of multi-objective optimization for tlp considering hull-form and tendon system. Ocean Engineering 116, 142-156.

Kim, M. H., 1997. Charm3D Users Manual.

Lee, J. Y., Koo, B. J., Clauss, G., 2007. Automated design of a tension leg platform with minimized tendon fatigue damage and its verification by a fully coupled analysis. Ship Technology Research 54, 11-27.

Lee, J. Y., Lim, S. J., 2008. Hull form optimization of a tension-leg platform based on coupled analysis. In: Proceedings of the 18th International Offshore and Polar Engiineering Conference, July 6-11, 2008, Vancouver, BC, Canada. 
Matos, V. L. F., Simos, A. N., Sphaier, S. H., 2011. Second-order resonant heave, roll and pitch motions of a deep-draft semi-submersible: Theoretical and experimental results. Ocean Engineering 38 (17), $2227-2243$.

Park, Y., Jang, B. S., Kim, J. D., 2015. Hull-form optimization of semi-submersible fpu considering seakeeping capability and structural weight. Ocean Engineering 104, 714-724.

Payne, G. S., Taylor, J. R. M., Bruce, T., Parkin, P., 2008. Assessment of boundary-element method for modelling a free-floating sloped wave energy device. Part 2: Experimental validation. Ocean Engineering $35(3), 342-357$.

Sivaraj, R., Ravichandran, D. T., 2011. A review of selection methods in genetic algorithm. International Journal of Engineering Science and Technology 3 (5).

Sugita, T., Suzuki, H., 2016. A study on tlp hull sizing by utilizing optimization algorithm. Journal of Marine Science and Technology 21 (4), 611-623.

Vannucci, P., 1996. Simplified optimal design of a tension leg platform (TLP). Structural Optimization 12 , $265-268$.

Williams, N., 2012. Optimized global sizing approach for gulf of mexico production semisubmersible. In: Proceedings of the 31st International Conference on Ocean, Offshore Mechanics and Arctic Engineering, OMAE2012-83114, Rio de Janeiro, Brazil.

Zhang, X., Bandyk, P., Beck, R. F., 2010. Time-domain simulations of radiation and diffraction forces. Journal of Ship Research 54 (2), 79-94.

Zhang, X., Song, X., Yuan, Z., You, Y., 2017. Global motion and airgap computations for semi-submersible floating production unit in waves. Ocean Engineering 141, 176-204. 